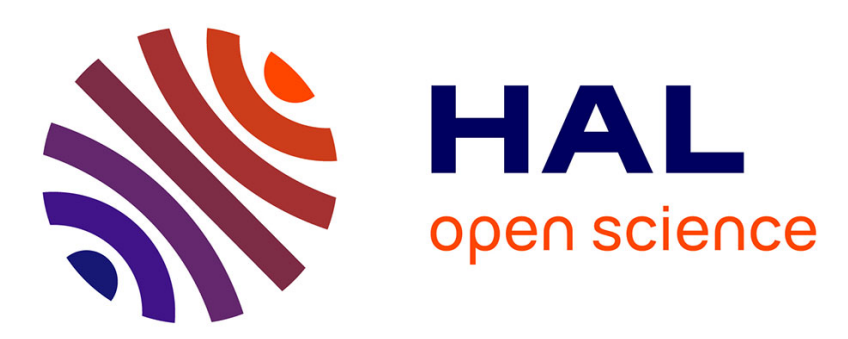

\title{
A Bayesian Approach for Thermal History Reconstruction in Basin Modeling
}

\author{
Andrea Licciardi, Kerry Gallagher, S. A. Clark
}

\section{To cite this version:}

Andrea Licciardi, Kerry Gallagher, S. A. Clark. A Bayesian Approach for Thermal History Reconstruction in Basin Modeling. Journal of Geophysical Research: Solid Earth, 2020, 125 (7), pp.e2020JB019384. 10.1029/2020JB019384 . insu-02926818

\section{HAL Id: insu-02926818 https://hal-insu.archives-ouvertes.fr/insu-02926818}

Submitted on 1 Sep 2020

HAL is a multi-disciplinary open access archive for the deposit and dissemination of scientific research documents, whether they are published or not. The documents may come from teaching and research institutions in France or abroad, or from public or private research centers.
L'archive ouverte pluridisciplinaire HAL, est destinée au dépôt et à la diffusion de documents scientifiques de niveau recherche, publiés ou non, émanant des établissements d'enseignement et de recherche français ou étrangers, des laboratoires publics ou privés. 


\section{JGR Solid Earth}

\section{RESEARCH ARTICLE \\ 10.1029/2020JB019384}

Key Points:

- Novel Bayesian approach for inverse thermal history modeling in sedimentary basins

- Joint inversion of multiple thermal indicator and porosity data from a borehole

- Heat flow history and erosion events are treated as primary unknowns

- Results are presented as probability distributions allowing a robust assessment of uncertainty

Supporting Information:

- Supporting Information S1

- Text S1

Correspondence to:

A. Licciardi,

andrea.licciardi@geoazur.unice.fr

Citation:

Licciardi, A., Gallagher, K., \& Clark, S. A. (2020). A Bayesian approach for thermal history reconstruction in basin modeling. Journal of Geophysical Research: Solid Earth,

125, e2020JB019384. https://doi.org/ 10.1029/2020JB019384

Received 13 JAN 2020

Accepted 8 JUN 2020

Accepted article online 13 JUN 2020

(C)2020. American Geophysical Union. All Rights Reserved.

\section{A Bayesian Approach for Thermal History Reconstruction in Basin Modeling}

\author{
A. Licciardi ${ }^{1,2}$ (D), K. Gallagher ${ }^{1}$ (D), and S. A. Clark $^{3}$ \\ ${ }^{1}$ Géosciences, Université de Rennes 1, Rennes, France, ${ }^{2}$ Now at Geoazur, Université Côte d'Azur, Sophia Antipolis, \\ France, ${ }^{3}$ Equinor Research, Trondheim, Norway
}

\begin{abstract}
We present a novel method for the joint inversion of thermal indicator data (vitrinite reflectance and apatite fission track) and additional data (bottom-hole temperature and porosity) for thermal history reconstruction in basin modeling. A transdimensional and hierarchical Bayesian formulation is implemented with a reversible jump Markov chain Monte Carlo algorithm, with a 1-D transient thermal model. The objective of the inverse problem is to infer the heat flow history below a borehole given the data and a set of geological constraints (e.g., burial histories and physical properties of the sediments). The algorithm incorporates an adaptive, data-driven parametrization of the heat flow history, allows for automatic estimation of the relative importance of each data type and quantification of parameter uncertainties and trade-offs. Our approach deals with uncertainties on the imposed geological constraints in two ways. First, the amount of erosion and timing of an erosional event are treated as independent parameters. Second, uncertainties on compaction parameters and surface temperature history are directly propagated into the final solution. Synthetic tests show that porosity data can be used to reduce uncertainties on the amount of erosion. This work illustrates a truly probabilistic analysis of the trade-off between the magnitude of erosion and variations in heat flow histories which is key in basin modeling. The algorithm is then applied to real data from a well in the Barents Sea. Our algorithm can reconcile estimates of erosion from the thermal indicator and porosity data, which is a difficult and subjective task in basin modeling.
\end{abstract}

\section{Introduction}

The main objective of thermal history modeling (THM) is to constrain the thermal evolution of a sedimentary basin over geological time. This, combined with subsidence analysis, provides first-order constraints for geodynamic models of basin evolution (Allen \& Allen, 2005). Furthermore, THM is a key aspect of hydrocarbon exploration and resource assessment (Hantschel \& Kauerauf, 2009), as hydrocarbon generation (maturation) in sedimentary basins depends strongly on the temperature history of the source rock. Assuming that the depositional history and the thermophysical properties of the sediments are known, the evolution of temperatures in a basin is controlled by temporal variations in boundary conditions, that is, heat flow at the base of the evolving basin and the temperature history at the Earth's surface. Once these are specified, it is then straightforward to predict the temperatures over time at a specific location in a basin by solving some form of the heat transfer equation.

A variety of temperature-sensitive data are routinely collected during borehole drilling and have been classically used to calibrate thermal history models through forward and/or inverse modeling approaches (Gallagher \& Sambridge, 1992; Green \& Duddy, 2012; Ketcham, 2005; Lerche, 1988; Schneider \& Issler, 2019; Waples et al., 1992a, 1992b). Such data are usually referred to as thermal indicator (TI) data (e.g., Waples, 1992b). These include, but are not limited to, vitrinite reflectance (VR), fission track analysis, and $\mathrm{U}-\mathrm{Th} / \mathrm{He}$ dating of apatite or zircon, illite-smectite transformation ratio, organic transformations (e.g., sterane/hopane isomerization and steroid aromatization), fluid inclusions, pollen translucency, and Rock-Eval parameters (e.g., $T_{\max }$ ) (e.g., McCulloh \& Naeser, 1989). Although each of these data type has its distinctive characteristic and range of sensitivity/applicability, they all share a dependence on temperature history, which makes them important as constraints in the context of THM in basin modeling (see Gallagher, 1998, for details about the general temperature dependence of TI data). 
From the inverse modeling perspective, the problem is to find a heat flow model, or preferably an ensemble of models, that is consistent with measured calibration data (e.g. TI, bottom-hole temperature (BHT), and porosity), allowing for uncertainties in the data and input parameters. Furthermore, we want to identify which parts of the heat flow history are well resolved, or not, by the calibration data. As stated above, this requires knowledge of the burial histories of the sediments, their physical properties and a surface temperature history. However, many of these input parameters (e.g., thermal conductivity, compaction parameters, paleosurface temperatures, and reaction kinetics) are not known reliably but are often fixed to constant values based on noisy measurements or previous experience. Additionally, uncertainties in burial histories will add to the uncertainties in the retrieved models. In particular, erosion can play a crucial role in THM, as the magnitude directly affects the temperatures experienced by sediments over geological time, through a modification of the burial history. Overall, then, quantification of the various uncertainties in THM is a key aspect in the interpretation of model results, but to our knowledge this has not yet been addressed directly with probabilistic methods.

Inverse THM has been previously carried out through linearized, derivative-based methods (Gallagher \& Sambridge, 1992; Gallagher, 1998; Lerche, 1988). These methods suffer from well-known limitations. First, a single best fit or optimal solution usually fails to capture the complexity of the problem. Due to nonlinearity between temperature and TI data (Gallagher \& Sambridge, 1992; Lerche, 1988) and the strong nonuniqueness of the problem (different temperature histories can produce similar fit to the data), there is a significant risk of becoming stuck in a local minima and missing alternative solutions. Second, implementing iterative linearized inversion methods requires a starting model, the choice of which can influence the final solution, again often due to the presence of local minima. Finally, although parameter sensitivity analysis can be performed (e.g., Gallagher, 1998; Nielsen, 1996; Waples et al., 1992b) a formal quantification of uncertainties and parameter trade-offs is more difficult.

One way to overcome these limitations is to cast the inverse problem in a Bayesian framework. The aim is to estimate probability distributions to characterize the ensemble of possible models that are consistent with the input calibration data. This can be achieved with Markov chain Monte Carlo (McMC) algorithms, a sampling method, which avoids the need to calculate derivatives and has proven to be effective for parameter inference and uncertainty quantification (see Gallagher et al., 2009, for a review in the context of geosciences). An extremely flexible implementation of the McMC algorithm called the reversible jump McMC (rjMcMC, Green, 1995, 2003) has gained popularity in many areas of Earth sciences thanks to its data-driven inference about model dimensionality and complexity (see section 1.1).

In the context of THM, Ferrero and Gallagher (2002) applied a fixed dimension McMC algorithm to invert VR data for heat flow history, as did Nielsen et al. (2017) to calibrate VR kinetic parameters for Basin $\% R_{o}$. Gallagher (2012) presented the first application of the rjMcMC algorithm for the inversion of multiple TI data, although this approach deals only with temperature histories directly, rather than burial and heat flow histories.

In this work, we introduce a newly developed rjMcMC algorithm for THM. The algorithm is specifically designed in the context of basin modeling in order to reconcile different data types commonly acquired in boreholes, paying particular attention to uncertainty quantification on the retrieved heat flow models. The code was developed and tested with vitrinite reflectance (VR) and apatite fission track (AFT) as these are amongst the most commonly used TI data in THM (Waples, 1994). In addition, we include BHT data as these represent direct measurements of temperature at present-day collected at a given borehole location. The algorithm can easily be adapted to incorporate additional TI data types (as those mentioned earlier), to exploit different sensitivities to temperature and time. The only requirements are the availability of an appropriate predictive model (e.g., thermally activated diffusion or chemical reaction progress) and some way to quantify the difference between the observed and predicted values (misfit/likelihood function) for the given data type.

The algorithm also allows us to treat the amount of eroded section and timing of erosional events as unknowns. When doing this, we show the benefit of inverting porosity (or a porosity proxy, such as sonic velocity) data together with TI data to reduce the nonuniqueness of the solution. Additionally, to account for uncertain input modeling parameters (e.g., compaction constants and paleosurface temperatures), we 
adopt a resampling approach to map such uncertainties into the final solution. We first present the methodology, and then demonstrate its application to synthetic and real data.

\subsection{Bayesian Approach and rjMcMC}

In a Bayesian framework, we deal with probability distributions relevant to the model parameters dependent on the observations. More formally, this is the posterior probability distribution (PPD) of the model parameters, given the data, and is expressed in Bayes' rule:

$$
p(\mathbf{m} \mid \mathbf{d}, \mathscr{I}) \propto p(\mathbf{m} \mid \mathscr{I}) p(\mathbf{d} \mid \mathbf{m}, \mathscr{I}),
$$

where $p()$ indicates probability density, $A \mid B$ indicates conditional probability (i.e., the probability of $A$ given $B$ ), $\mathbf{d}$ is the vector of observed data, and $\mathbf{m}$ is the vector of model parameters. The term $\mathscr{I}$ indicates information common to all models, and, in this work, it can include geological assumptions and hypotheses as well as the specific details of a particular geodynamic model (e.g., the anticipated form of the heat flow history). The term $p(\mathbf{m} \mid \mathbf{d}, \mathscr{I})$ is the target PPD of the model parameters, $p(\mathbf{m} \mid \mathscr{I})$ represents the prior probability distribution on the model parameters, or what we believe is reasonable in the absence of any observations. Finally, $p(\mathbf{d} \mid \mathbf{m}, \mathscr{I})$ is the likelihood, that is, the probability of observing the measured data values given a certain set of model parameter values, or a measure of how well the predictions from that model agree with the observations. The likelihood increases as the model fits the data better. In the Bayesian framework, the likelihood effectively updates the prior information, transforming it to the posterior. If the data provide no constraints for some parts of the model, then when estimating the posterior, we should recover the prior for those parts of the model.

In the context of THM, an analytical expression for the PPD is impossible to obtain and we need to rely on numerical methods in order to sample the unknown PPD. The rjMcMC algorithm (Green, 1995, 2003) allows to make inference on both model parameters and model complexity at the same time by treating the number of parameter $(n)$ itself as an unknown. Several authors have shown the advantages of transdimensional inference (see Sambridge et al., 2013, for a review). Examples of successful applications of this type of algorithm can be found in seismology (Bodin, Sambridge, et al., 2012; Piana Agostinetti et al., 2015; Ravenna \& Lebedev, 2017), mixture modeling in geochronology (Jasra et al., 2006), geospatial analysis (Bodin, Salmon, et al., 2012; Licciardi et al., 2020), exploration geophysics (Ray et al., 2016), stratigraphy (Charvin et al., 2009), magnetotellurics (Mandolesi et al., 2018; Xiang et al., 2018), geoacoustics (Dettmer et al., 2010; Dosso et al., 2014), and borehole geophysics (Reading \& Gallagher, 2013).

A key concept in transdimesional inference is its implicit natural parsimony: given two models with the same likelihood, the simplest (i.e., with less parameters) is preferred (Malinverno, 2002; Mackay, 2003). This gives rise to an adaptive parametrization, which is solely dictated by the information contained in the data. This is an important feature of our algorithm as it allows the complexity of the heat flow model (the number of points in the heat flow history, see section 2.4) to be directly inferred from the data, thus reducing any potential user-induced bias.

The main output of the algorithm is a transdimensional PPD, which contains all the information about the model parameters but this is difficult to visualize in practice. However, as the models sampled by the algorithm are distributed according to the PPD, the sample histogram of a parameter approximates its marginal PPD, which is easy to visualize. Different statistics (e.g., mean and standard deviation) can be computed directly from the frequency distribution of each parameter.

\section{Method}

A workflow of the algorithm presented in this work is sketched in Figure 1. Each of the components of the algorithm is discussed in the following sections.

\subsection{Input Data}

The algorithm as we have coded it can deal with four different types of input data. In the following, we differentiate between TI data types (VR, AFT) and porosity data which will be used to constrain the amount of erosion. We also use direct temperature measurements (BHT), which provide constraints on the present day thermal state of the well. We use the terms calibration data or input data to refer to the whole ensemble of TI, 


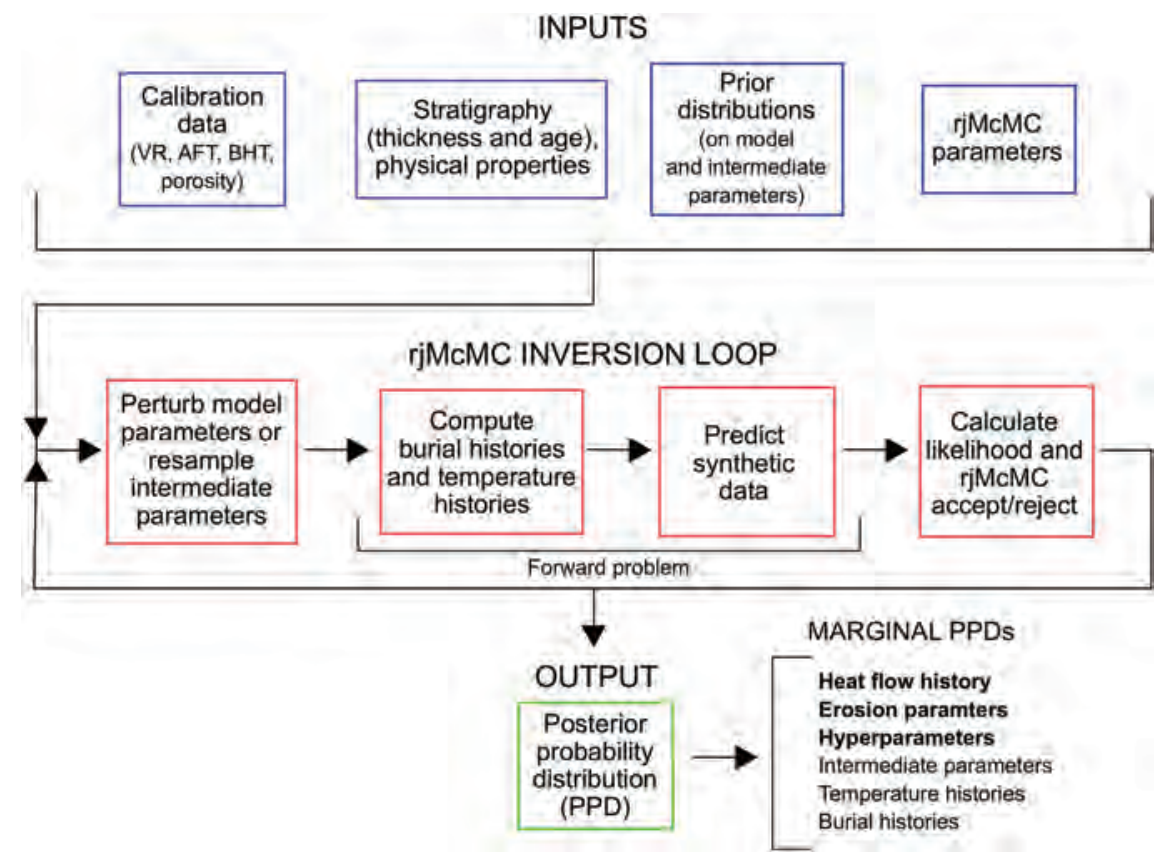

Figure 1. Simplified workflow of the algorithm. Under marginal PPDs, bold face font indicates the model parameters, estimated during the inversion. Intermediate parameters are the compaction constants and the surface temperature history, and these are resampled during the inversion. Please refer to the text for more details.

BHT, and porosity data which are jointly inverted by our algorithm. In all cases, the data are typically collected over a range of different depths in a borehole, which can be different for each data type.

VR data have a long history of applications in thermal history reconstructions (McCulloh \& Naeser, 1989). They have mostly been used as a proxy for maturity and have proven to be primarily sensitive to the maximum temperature experienced by the host rock, with minimal information on timing (Waples, 1994). In general, VR data are presented as a mean value of $R_{0}$ in percent and an associated error. In contrast, AFT data provide both time and temperature information (e.g., Gallagher et al., 1998; Green \& Duddy, 2012; Malusà \& Fitzgerald, 2018). A typical AFT data set is comprised of a number of single grain ages, track length measurements and some form of grain compositional information. As TI predictive models typically depend on both temperature and time, we first need to compute the temperature histories for a given heat flow history and stratigraphy (details in section 2.2).

BHT data are measurements of present day downhole temperature and are generally corrected for nonsteady state effects due to drilling mud circulation. These data provide constraints on the present-day heat flow.

Finally, we can incorporate porosity data in our joint inversion scheme. Under the assumption of simple mechanical compaction, porosity data (or a proxy such as sonic-log velocities) can be used to estimate the amount of net erosion at a borehole location (Corcoran \& Doré, 2005). In our approach, observed porosity data (fractional total porosity and error) are compared to predicted porosity values as the amount of erosion is allowed to vary in the inversion (inducing variations in the burial and compaction histories of the sediments).

\subsection{Forward Problem}

Solving the forward problem means making predictions given a combination of burial, heat flow, and surface temperature histories to allow comparison to calibration data (Figure 1).

For TI data, we use a similar general approach described in Gallagher and Sambridge (1992). First, the temperature histories of selected sedimentary layers are obtained by solving some form of the heat equation (described below). Second, the temperature history is used to predict TI data by solving the corresponding predictive equations. 
In contrast to the 1-D approximation of the heat equation in steady state (Gallagher \& Sambridge, 1992), here we use a 1-D transient formulation. The 1-D approach is considered valid in sedimentary basins where dominantly vertical heat transfer is expected. This approach would not be valid if there is significant lateral heat transfer, particularly related to localized fluid flow involving anomalously hot or cold fluids, or major lateral thermal conductivity contrasts (e.g., salt diapirs). A steady state approximation should be valid where the thermal re-equilibration timescale of the sedimentary pile is rapid ( $\approx 10^{4}-10^{6}$ years) compared to the long-term evolution of the basin $\left(\approx 10^{7}-10^{8}\right.$ years $)$. This is generally the case when the length scale of the problem (i.e., the depth to the lower boundary heat flow condition) is just a few kilometers, such as at the base of the sedimentary pile, as opposed to the thickness of the crust or the lithosphere. For generality however, we prefer to adopt the transient formulation as this will capture the steady state models when the assumption is valid and also allows the depth to the lower boundary condition to be specified well below the sedimentary pile, if desired. We also allow for heat production and advective heat transfer (related to sedimentation/erosion), but not for vertical fluid flow.

The governing equation we adopt is then

$$
\frac{\partial(\rho C T)}{\partial t}=\frac{\partial}{\partial z}\left(k \frac{\partial T}{\partial z}\right)-u \rho C \frac{\partial T}{\partial z}+H,
$$

where $T$ is temperature $\left({ }^{\circ} \mathrm{C}\right), t$ is time $(\mathrm{s}), z$ is depth $(\mathrm{m}), k$ is thermal conductivity $\left(\mathrm{W} \mathrm{m}^{-1} \mathrm{~K}^{-1}\right), \rho$ is density $\left(\mathrm{kg} \mathrm{m}^{-3}\right)$, and $C$ is specific heat capacity $\left(\mathrm{J} \mathrm{K}^{-1} \mathrm{~kg}^{-1}\right), u$ is velocity $\left(\mathrm{m} \mathrm{s}^{-1}\right)$, and $H$ is heat production $\left(\mathrm{W} \mathrm{m}^{-3}\right)$. The thermal diffusivity $\left(\kappa, \mathrm{m}^{2} \mathrm{~s}^{-1}\right)$, is further defined as

$$
\kappa=\frac{k}{\rho C}
$$

The physical properties defined above are lithology and porosity dependent, and will vary with compaction. To solve Equation 2, with depth dependent parameters, we use a Lagrangian finite difference scheme, as described in the supporting information (SI) material.

In basin modeling, stratigraphic data from boreholes (age, thickness, lithology, presence of unconformities, and inferred thickness of missing section) are used to reconstruct the burial histories of each sedimentary layer. Here, burial histories are calculated assuming linear deposition of the solid grain component in each stratigraphic unit, with the solid grain component estimated using standard decompaction methods (e.g., Gallagher, 1989).

We assume an exponential reduction of porosity with depth following:

$$
\Phi(z)=\Phi_{0} \exp (-c z)
$$

where $\Phi_{0}$ and $c$ are porosity at the surface and compaction factor, both depending on lithology. In this case, the solid grain component (or thickness) for a given sedimentary layer between depths $z_{1}$ and $z_{2}$ is given as

$$
V_{s}=z_{2}-z_{1}+\frac{\Phi_{0}}{c}\left(\exp \left(-c z_{2}\right)-\exp \left(-c z_{1}\right)\right)
$$

Once $\Phi(z)$ is calculated for a given $t$, an average value $\hat{\Phi}$ is used to compute the bulk thermal conductivity, $k_{b}$, for each formation (layer), using a geometric mean,

$$
k_{b}=k_{f}^{\hat{\phi}} \prod_{i=1}^{N_{l}} k_{m(i)}^{\Psi_{i}}
$$

where $N_{l}$ is the number of different lithologies in the formation, $k_{f}$ is the pore fluid thermal conductivity, $k_{m(i)}$ is the matrix thermal conductivity of the $i$ th lithology, and $\Psi_{i}$ is the proportion of that lithology in the formation. The other thermophysical properties (heat capacity, density, and heat production) are calculated using the arithmetic mean equivalent of the thermal conductivity equation above. 
Having defined all the appropriate thermophysical parameters, we solve Equation 2 to obtain thermal histories for each stratigraphic layer and/or TI sample and these are used with the appropriate models to make predictions. For VR data, we use the model of Sweeney and Burnham (1990) with default kinetic parameters for Easy\% $R_{o}$. For AFT data the multicompositional algorithms of (Ketcham et al., 1999, 2007) are used. The predictions can then be compared to the TI observations. Finally, the calculated values of temperature at present day are directly compared with the observed BHT data at their specific depths. When allowing for variable magnitudes of erosion in the inverse modeling, the porosity structure of the sedimentary column will also vary and then the thermophysical parameters need to be updated accordingly each time this occurs. The predicted porosity profile is then compared to the observed porosity data.

\subsection{Uncertain Intermediate Parameters}

As pointed out by Gallagher (2012), uncertainties in predictive models for TI data are often nonnegligible, arising from noise in the calibration data and the typically empirical nature of the models proposed. Moreover, the forward problem involves various stages, including burial history and temperature calculations, in addition to the aforementioned predictive models for TI data. Each stage also has uncertainty inherent in its parameters due to the fact the parameters are not measured directly and/or the calibrations are based on values that incorporate measurement error. We refer to these input parameters as "intermediate," as they are not of direct interest but are required to solve the problem. Ideally, we want to deal with uncertain intermediate parameters, rather than simply choosing fixed values. A possible way to address this issue is to resample the appropriate intermediate parameters involved in each stage, given a range of plausible values (expressed as prior distributions, Figure 1). This also provides a way of mapping the uncertainties into the final solution. In the general case, the number of uncertain quantities rapidly grows, making such a resampling approach more computationally expensive.

In particular, values of thermal conductivity (which are usually uncertain in nature) could be handled with such resampling approach. However, as discussed in Gallagher (2002), TI data are mainly sensitive to the average temperature gradient at any time, which is equivalent to the ratio of heat flow and average thermal conductivity. Although the absolute values of thermal conductivity tend to trade-off with the absolute values of the estimated heat flow, the overall form of the estimated heat flow history does not change appreciably. For this reason, here we have decided not to use a resampling approach for thermal conductivity or thermal diffusivity (directly proportional to the thermal conductivity and may affect timescales of heat transfer) but this is straightforward to implement if desired.

Instead, we focus the attention on two intermediate parameters involved in the porosity prediction and in temperature calculations: compaction parameters $\left(\Phi_{0}\right.$ and $c$ in Equation 4$)$ and surface temperature history (the upper boundary condition when solving Equation 2). This choice is intended just as a proof of concept, as in principle it is possible to investigate the contribution of all uncertain intermediate parameters to the final solution using a similar approach.

\subsection{Model Parametrization}

In our approach, the main model parameters are represented by a set of $n$ heat flow $(Q)$ nodes in time (Figure 2a). The number of $Q$ time nodes is an unknown itself and is at the core of our adaptive parametrization. Points are randomly added or removed along the sampling according to a birth-death strategy (see SI for more details). A continuous function $Q(t)$ is obtained by linear interpolation of the values at the nodes on a discretized grid, which constitutes the lower boundary condition to solve Equation 2 . Two additional parameters $Q_{\text {pres }}$ and $Q_{\text {tmax }}$ are also inserted at fixed time positions equal to present day $(t=0)$ and an upper time limit $\left(t=t_{\max }\right)$, for example, the oldest stratigraphic age, respectively. So, the vector of model parameters involving $Q$ has variable dimensions $2 n+2$ and can be written as $\mathbf{Q}=\left[t_{1}, \ldots, t_{N}, Q_{1}, \ldots, Q_{N}, Q_{\text {pres }}, Q_{\text {tmax }}\right]$.

Our choice for parametrizing an erosional event is illustrated in Figure $2 \mathrm{~b}$. We assume that the duration spanned by the unconformity is known (defined by $t_{U 1}$ and $t_{U 2}$ ) and that within the unconformity a certain thickness of sediment is deposited and then eroded. An erosional event is therefore defined by the amount of section $\Delta E$ removed between the time interval delimited by $t_{\text {start }}$ and $t_{\text {end }}$. Therefore, this implicitly requires the deposition of the same amount of sediments between $t_{U 1}$ and $t_{\text {start }}$. Here, we treat $\Delta E$, $t_{\text {start }}$ and $t_{\text {end }}$ as unknowns, with $t_{U 1}$ and $t_{U 2}$ fixed. In this way, we can write the vector of parameters concerning erosion as $\mathbf{E r}=\left[\Delta E, t_{\text {start }}, t_{\text {end }}\right]$, with $t_{U 1}>=t_{\text {start }}>t_{\text {end }}>=t_{U 2}$. 
(a)

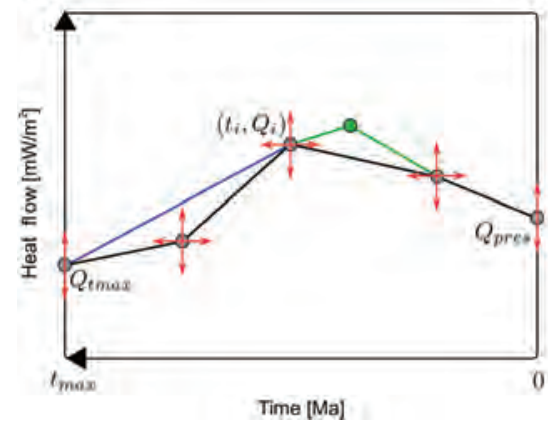

(b)

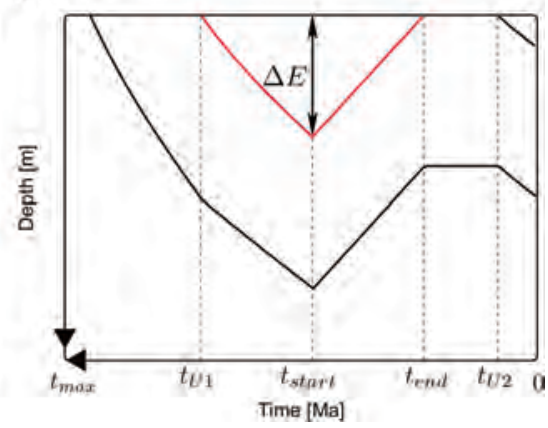

Figure 2. Model parametrization used in the algorithm. (a) The heat flow history is defined by a series of nodes (heat flow and time) and heat flow values are obtained by linear interpolation between the nodes. Possible perturbations of each point are indicated by red arrows. Green and blue lines indicate possible configuration of the heat flow history after birth (add a node) and death (remove a node), respectively. (b) Parametrization of an erosional event, the parameters $\mathbf{E r}=\left[\Delta E, t_{\text {start }}, t_{\text {end }}\right]$ are treated as unknowns in the inversion algorithm as can be varied as in (a). The red lines indicate the burial path of a missing sedimentary section. See the main text for more details.

In our algorithm, the roles of $T_{s}(t)$ (surface temperature history) and compaction parameters are investigated by sampling their values from the corresponding prior distributions as part of the iterative inversion process. $T_{s}(t)$ is specified as a sequence of $N_{T}$ points in time, which are linearly interpolated and serve as the upper boundary condition in Equation 2 . The compaction parameters ( $\Phi_{0}$ and $c$ in Equation 4$)$ describes the shape of the compaction curve for each of the $N_{l}$ lithologies and influence the estimated thickness of eroded section and the values of porosity dependent thermophysical parameters.

Finally, the algorithm follows a Hierachical approach (Malinverno \& Briggs, 2004) in which, the observed errors for each data type (VR, AFT, BHT, and porosity data) are rescaled by a set of hyperparameters. We define a vector of such hyperparameters, $\mathbf{h}=\left[\mathbf{h}_{v r}, h_{b h t}, h_{p o r}\right]$, where $\mathbf{h}_{v r}=\left[h_{v r, 1}, \ldots, h_{v r, N_{v r}}\right]$ and $N_{v r}$ is the number of VR data points. Thus, we use one hyperparameter for each VR data point but just one each for the BHT and porosity data. The importance of these hyperparameters in a joint inversion scheme is discussed in section 2.5.3.

To summarize, the full vector of model parameters is $\mathbf{m}=[n, \mathbf{Q}, \mathbf{E r}, \mathbf{h}]$. In addition, the intermediate parameters, $T_{s}(t), \Phi_{0}$ and $c$ are resampled during the inversion process (Figure 1).

\subsection{Implementation}

\subsubsection{Sampling Strategy and Acceptance Probability}

The rjMcMC is a generalization of the Metropolis-Hasting algorithm (Hastings, 1970; Metropolis et al., 1953), which can be summarized as follows: After having chosen the prior distributions for each model parameter (including $n$ ), an initial model $\left(\mathbf{m}_{0}\right)$ is directly drawn from these prior distributions, the forward problem is solved and the likelihood, $p(\mathbf{d} \mid \mathbf{m}, \mathscr{I})$ is calculated for this model.

At each iteration, a new, or candidate, model, $\mathbf{m}_{c}$ is selected probabilistically by perturbing $\mathbf{m}_{0}$. For $\mathbf{m}_{c}$, the forward problem is also solved and its likelihood calculated. The candidate model is then accepted with a probability $\alpha$ given by

$$
\alpha=\min \left[1, \frac{q\left(\mathbf{m}_{0} \mid \mathbf{m}_{c}\right) p\left(\mathbf{m}_{c} \mid \mathscr{I}\right) p\left(\mathbf{d} \mid \mathbf{m}_{c}, \mathscr{I}\right)}{q\left(\mathbf{m}_{c} \mid \mathbf{m}_{0}\right) p\left(\mathbf{m}_{0} \mid \mathscr{I}\right) p\left(\mathbf{d} \mid \mathbf{m}_{0}, \mathscr{I}\right)}|\mathbf{J}|\right]
$$

where $p(\mathbf{m} \mid \mathscr{I})$ is the prior distribution, $p(\mathbf{d} \mid \mathbf{m}, \mathscr{I})$ is the likelihood, $q\left(\mathbf{m}_{c} \mid \mathbf{m}_{0}\right)$ is the proposal distribution (the probability of obtaining a candidate $\mathbf{m}_{c}$ when the current model is $\mathbf{m}_{0}$ ), and $|\mathbf{J}|$ is the determinant of the Jacobian matrix of the transformation from $\mathbf{m}_{0}$ to $\mathbf{m}_{c}$, which is needed for transformations that involve a change in dimension (transdimensional), although the algorithm can be designed so that its value is always unity. If $\mathbf{m}_{c}$ is accepted, it becomes the current model vector $\mathbf{m}_{0}$. The process proceeds iteratively for a number of total iterations $\left(N_{t o t}\right)$ and requires a number of burn-in iterations $\left(N_{\text {burn }}\right)$ to properly converge to the PPD. Models are saved for posterior inference only for the iterations after the burn-in phase. 
The selection of $\mathbf{m}_{c}$ from $\mathbf{m}_{0}$ is at the core of the algorithm. A set of rules needs to be carefully designed for each specific inverse problem (Piana Agostinetti \& Bodin, 2018). These rules make up what is called the recipe of our implementation. The recipe also determines the performance of the algorithm in terms of efficiency of the exploration of the model space (Mandolesi et al., 2018). Common practice is to design an algorithm for which the acceptance rate of proposed models using the Metropolis-Hasting rule is around 20-50\% (Robert \& Casella, 2004). In this work, we follow the approach described in Mosegaard and Tarantola (1995) and in the Appendix A of Piana Agostinetti and Malinverno (2010), which allows us to simplify Equation 7 to

$$
\alpha=\min \left[1, \frac{p\left(\mathbf{d} \mid \mathbf{m}_{c}, \mathscr{I}\right)}{p\left(\mathbf{d} \mid \mathbf{m}_{0}, \mathscr{I}\right)}\right]
$$

which simply involves the likelihood ratio between candidate and current models. For $\mathbf{Q}$, a summary of possible perturbations of $\mathbf{m}_{0}$ (including the number of points) is sketched in Figure 2a. More details on this and a full description of the recipe of our implementation can be found in the SI.

\subsubsection{Likelihood Function}

The likelihood plays a fundamental role in determining which samples will be accepted into the final distribution of models (Figure 1). In this work, we use two types of likelihood function and we deal with their log values for numerical stability. For AFT data, we use the log likelihood function described in Gallagher (1995), which uses the individual track counts and track lengths as data, while for VR, BHT, and porosity data we use a Gaussian distribution for the likelihood:

$$
p(\mathbf{d} \mid \mathbf{m})=\frac{1}{\sqrt{(2 \pi)^{N}\left|\mathbf{C}_{d}\right|}} \exp \left(-\frac{\left(\mathbf{d}_{p r e}-\mathbf{d}\right)^{T}\left(\mathbf{C}_{d}^{-1}\right)\left(\mathbf{d}_{p r e}-\mathbf{d}\right)}{2}\right)
$$

where for each data type, $\mathbf{d}$ is the vector of observed data, $\mathbf{d}_{\text {pre }}$ contains the data predicted by the current model, $N$ is the length of the data vector, and $\mathbf{C}_{d}$ is the covariance matrix of the data errors. We consider each data type to be independent from the others so that the total likelihood for each model is just the product of the individual likelihoods for each data type (or the sum of the log likelihoods).

\subsubsection{Hierarchical Approach}

The matrix $\mathbf{C}_{d}$ in Equation 9 describes the magnitude and correlation of the data noise. It determines the range of acceptable solutions in terms of data fit as the predictions of the accepted models should fall within the range given by the observed data and their uncertainties. For example, the inferred number of parameters $(n)$ in a model strictly depends on the data noise (Bodin, Sambridge, et al., 2012; Piana Agostinetti \& Malinverno, 2010). As the measurement errors decrease on each sample, models with higher complexity (which produce a better fit to the data) will be more likely to be accepted by the algorithm. In practice, if the magnitude of data error is fixed in the inversion this will determine a priori the model complexity required to fit the data. However, in many situations (and THM is not an exception) precise estimates of measurement errors are difficult to obtain. Unreliable or perhaps erroneous estimates of the data noise may produce a bias in the resulting model complexity and could lead to overfitting of the data. In the Hierarchical Bayesian approach (Bodin, Sambridge, et al., 2012; Malinverno \& Briggs, 2004) the magnitude of data error is treated as an unknown and is directly inferred from the data. This is implemented by scaling the covariance matrix for the $i-t h$ data set, $\mathbf{C}_{d, i}^{*}$ with the hyperparameters $h_{i}$ described in section 2.4 , in such a way that

$$
\mathbf{C}_{d, i}=10^{h_{i}} \times \mathbf{C}_{d, i}^{*}
$$

These hyperparameters are effectively treated as model parameters to be inferred from the input data by the rjMcMC sampling. They provide an estimate of the posterior uncertainties on data errors and simultaneously act as weighting factors of different data types (Bodin, Salmon, et al., 2012; Licciardi et al., 2020). This is of key importance in our joint inversion scheme, as the relative importance of each data type (VR, AFT, BHT, and porosity data) is usually not known a priori. In addition, the combined data may be incompatible (i.e., fitting all the data to within their prescribed error ranges may not be possible). The hyperparameters effectively control the relative influence of each data type by increasing (decreasing) its observed errors (by scaling $\mathbf{C}_{d, i}^{*}$ ) and therefore by decreasing (increasing) its relative importance in the joint inversion. 
Table 1

Summary of the Prior Distributions for $\boldsymbol{m}$ and Intermediate Parameters Used for the Synthetic Test and the Real Data Application

\begin{tabular}{lcc}
\hline $\begin{array}{l}\text { Model } \\
\text { parameter }\end{array}$ & $\begin{array}{c}\text { Synthetic } \\
\text { data }\end{array}$ & $\begin{array}{c}\text { Real } \\
\text { data }\end{array}$ \\
\hline$n$ & $U[1,40]$ & $U[1,30]$ \\
$t(\mathrm{Ma})$ & $U(0,120)$ & $U(0,250)$ \\
$Q\left(\mathrm{~mW} \mathrm{~m}^{-2}\right)$ & $U(0,120)$ & $U(20,200)$ \\
$Q_{\text {pres }}\left(\mathrm{mW} \mathrm{m}^{-2}\right)$ & $U(0,120)$ & $U(20,200)$ \\
$Q_{\text {tmax }}\left(\mathrm{mW} \mathrm{m}^{-2}\right)$ & $U(0,120)$ & $U(20,200)$ \\
$\Delta E(\mathrm{~m})$ & $U(0,4000)$ & $U(0,4000)$ \\
$t_{\text {start }}(\mathrm{Ma})$ & $U(20,40)$ & $U(30,75)$ \\
$t_{\text {end }}(\mathrm{Ma})$ & $U(10,20)$ & $U(2,10)$ \\
$\mathbf{h}$ & $U(-1,1)$ & $U(-1,1)$ \\
\hline Intermediate & Synthetic & Real \\
parameter & data & data \\
\hline$T_{S}(0)\left({ }^{\circ} \mathrm{C}\right)$ & $\mathcal{N}\left(20.0,2.0^{2}\right)$ & Fixed \\
Sand. $\Phi_{0}$ & $\mathcal{N}\left(0.49,0.05^{2}\right)$ & $\mathcal{N}\left(0.49,0.1^{2}\right)$ \\
Silt. $\Phi_{0}$ & & $\mathcal{N}\left(0.56,0.1^{2}\right)$ \\
Shale $\Phi_{0}$ & & $\mathcal{N}\left(2.7,0.6^{2}\right)$ \\
Sand. $c\left(10^{-4} \mathrm{~m}^{-1}\right)$ & $\mathcal{N}\left(2.6,0.2^{2}\right)$ & $\mathcal{N}\left(3.9,0.9^{2}\right)$ \\
Silt. $c\left(10^{-4} \mathrm{~m}^{-1}\right)$ & & $\mathcal{N}\left(6.3,0.6^{2}\right)$ \\
Shale $c\left(10^{-4} \mathrm{~m}^{-1}\right)$ & & \\
\hline
\end{tabular}

Note. See section 2.4 for a description of model and intermediate parameters.
For AFT data, an effective implementation of the hierarchical approach is difficult given the form of the likelihood function (see Gallagher, 1995), which does not have a readily scalable parameter like $\mathbf{C}_{d}$. In this work, the Hierarchical approach is implemented using one hyperparameter for each VR data point and a single one for BHT and porosity data. In this way, we use AFT data as a reference and the hyperparameters quantify the relative importance of any other data type with respect to that reference.

\subsubsection{Prior Distributions}

In any Bayesian approach, the prior distributions play a key role in estimating the posterior as they define the possible values of the parameters to be sampled by the Markov chain. Working with prior distributions offers a flexible framework to define the level of prior knowledge on each model parameter. At the same time, prior distributions must be carefully chosen to avoid physically unrealistic models. A summary of the prior distributions used in this work is reported in Table 1. These distributions can be easily modified according to previous studies or any kind of scientific knowledge. When testing a new McMC algorithm, it is good practice to perform a prior-sampling test by setting the acceptance probability of Equation 8 to unity (Mandolesi et al., 2018; Piana Agostinetti \& Malinverno, 2010). This is equivalent to saying the data provide no information for the inversion. Then, the posterior distribution returned by the algorithm should be equal to the prior. The successful results of this test are reported in the SI.

\subsubsection{Parallel Tempering and rjMcMC Parameters}

To improve the efficiency of sampling models from complex (e.g., multimodal) posterior distributions, we embed the algorithm described in the previous section in a parallel tempering (PT) scheme (Falcioni \& Deem, 1999; Geyer, 1991). In PT, multiple chains are run with different values of a parameter $(T)$ that scales the likelihood by progressively flattening it as $T$ increases. Chains are allowed to swap their $T$ parameters, effectively improving the efficiency of model space exploration and accelerating convergence (Dosso et al., 2014; Ray et al., 2016; Sambridge, 2013). Models are collected from the chain with $T=1$ for posterior inference (see SI for more details). In all model runs present in this work, we use 240 chains of which 40 at $T=1$. $T$ values for the remaining chains are chosen randomly from a log-uniform distribution with $1 \leq T \leq 50$. Each chain proceeds for $N_{t o t}=5 \times 10^{5}$ iterations and a $T$ swap is proposed at each iteration. We start collecting models from the chains at $T=1$ after discarding the first half of the iterations $\left(N_{\text {burn }}=N_{\text {tot }} / 2\right)$ and we save the accepted models every 100 iterations (chain thinning) to avoid correlations between models. Therefore, after sampling $1.2 \times 10^{8}$ models, the final ensemble for posterior inference is based on $10^{5}$ models. These parameters need to be specified by the user and are referred as rjMcMC parameters in Figure 1.

\section{Application to Synthetic Data}

In this section, we use synthetic data generated from a specified $Q$ model and stratigraphy to assess the performance of the inversion algorithm. Here, we assumed one lithology for simplicity as the main aim is to assess the relationships between the major geological parameters (heat flow and erosion magnitude), without adding the complexity of variable lithologies. However, the approach we use can readily deal with multiple lithologies, as we demonstrate in the real data example. Details about the stratigraphic information of physical properties used in the test are given in the SI (Table S1).

We generated "perfect" TI data as a function of depth based on the thermal histories predicted with a constant $\left(40 \mathrm{~mW} \mathrm{~m}^{-2}\right) Q$ model at the base of the sediments and with $T_{s}(t)$ of $20^{\circ} \mathrm{C}$, also constant over time. The temperature and burial histories associated with the true $Q$ model are shown in Figures $3 \mathrm{a}$ and $3 \mathrm{~b}$. The burial history incorporates a single erosion event with the following true values: $\Delta E=2,000 \mathrm{~m}, t$ $s_{\text {start }}=30 \mathrm{Ma}, t_{\text {end }}=15 \mathrm{Ma}$. Using the predicted thermal histories, we generated synthetic AFT data (39 track lengths and 23 track counts) for 11 samples (see Gallagher, 1995, for more details on an approach to produce synthetic AFT data). Similarly, we produced synthetic VR data for 15 samples and 11 synthetic 
(a)

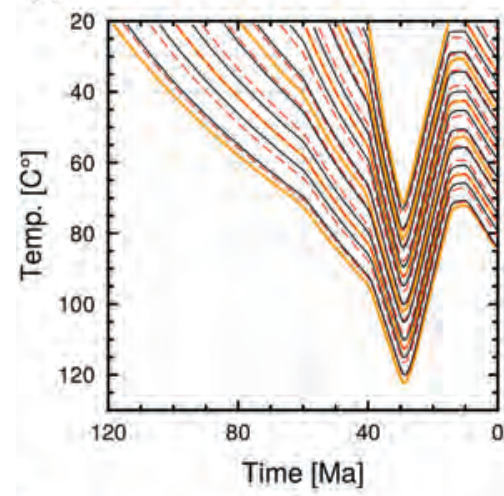

(c)

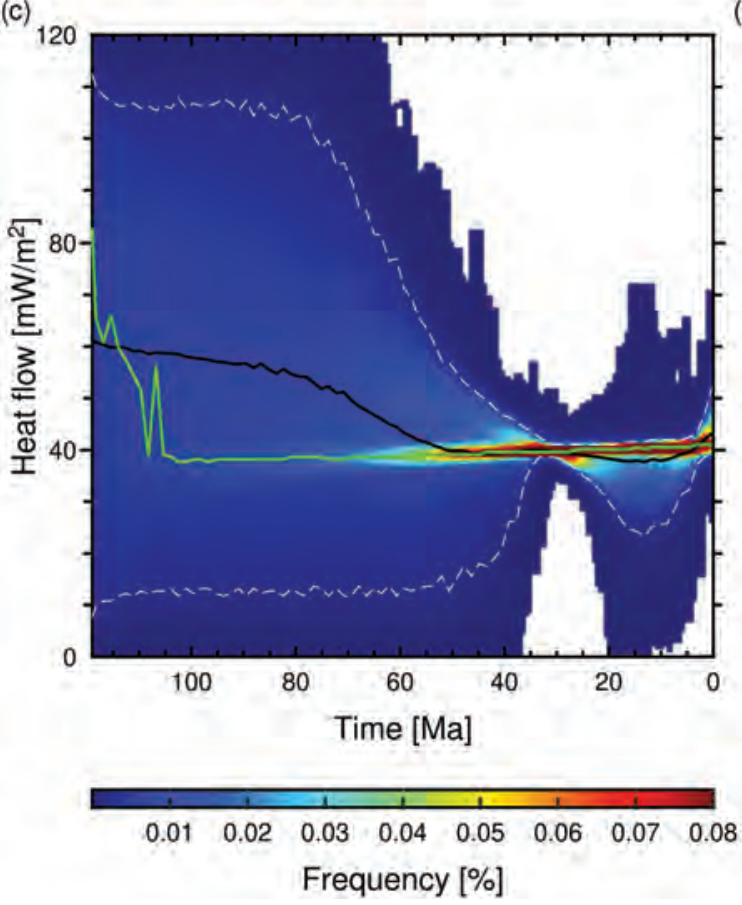

(b)

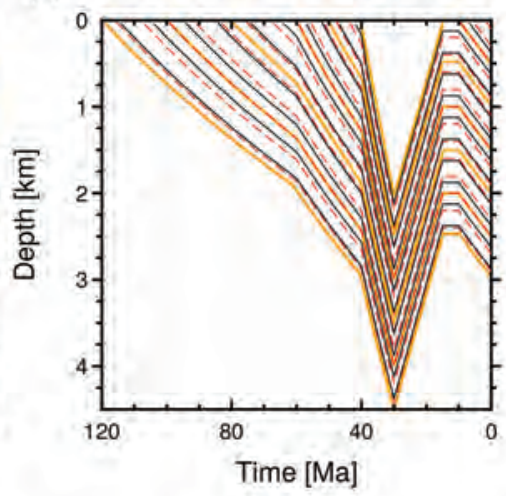

(d)

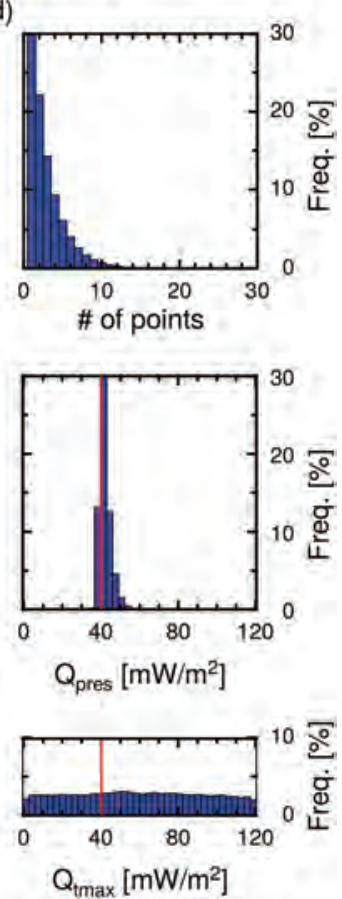

Figure 3. Results of the Synthetic Test 1. Synthetic data (AFT, VR, and BHT) are generated using a constant $\left(40 \mathrm{~mW} \mathrm{~m}^{-2}\right)$ heat flow model. These, together with porosity data, are incorporated in the inversion. (a) Temperature histories and (b) burial histories of the true model for stratigraphic layers (orange lines) VR samples (dashed red lines) and AFT samples (black lines). (c) Marginal posterior distribution of $Q(t)$ from interpolation between sampled nodes, with red to blue indicating more to fewer models. The black and green lines show the posterior estimates of the mean and mode $Q$ models, respectively. White dashed lines indicate $95 \%$ credible interval. (d) The three panels show the marginal posterior distribution for the number of nodes, $Q_{\text {pres }}$ and $Q_{t m a x}$. Red vertical lines indicate the values of the true model.

BHT data, both with added Gaussian noise with a standard deviation of $10 \%$ of the predicted value. Synthetic porosity data with $10 \%$ Gaussian noise were also produced given the calculated burial histories. Data noise correlation is not introduced in any of the data, so for the VR, BHT, and porosity data $\mathbf{C}_{d}$ is diagonal. Overall, the combined data can be regarded as representing a moderate to good quality data set.

The prior distributions described in Table 1 are used for this test. We ran two main inversions, the first in which porosity data were included (Synthetic Test 1) and the second in which they were not (Synthetic Test 2), the aim being to assess the role of the porosity data in constraining erosion. Prior to addressing that comparison, we consider the results of the first inversion, Synthetic Test 1, in terms of the other inversion parameters. These are shown in Figure 3, with a visual comparison of the predictions and the original data in the SI (Figure S2), together with the temperature and burial histories predicted by the posterior mean 


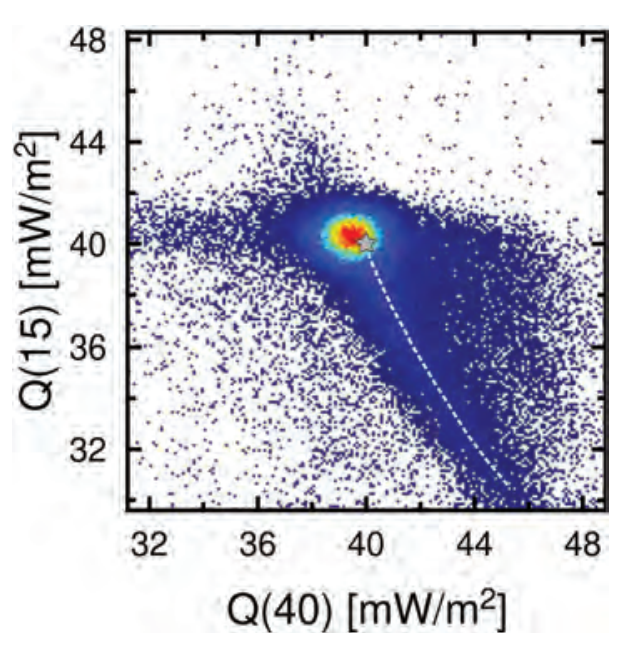

Figure 4. Results of the Synthetic Test 1. Posterior 2-D marginal for two heat flow points at $15 \mathrm{Ma}$ and $40 \mathrm{Ma}$. $Q(15)$ and $Q(40)$ are approximated by the values of $\mathrm{Q}$ closest to 15 and $40 \mathrm{Ma}$ in the interpolated $Q(t)$ for each model. The red to blue colors indicate higher to lower probability respectively. The gray star indicates the true parameter values. The white dashed line highlights a trend of negative correlation, which however has low probability. model (Figure S3). Note that in Figure 3c we plot the marginal PPD of the sampled $\mathbf{Q}$ after interpolation between nodes rather than the marginal PPD of the directly sampled nodes, because it is easier to interpret.

Using the $95 \%$ credible range as a guide, we see that the best constrained part of the model is around $30 \mathrm{Ma}$ (Figure 3c). As we would expect, this corresponds to the time of maximum temperature (Figure 3a), and in this case also to the time of maximum burial (Figure $3 \mathrm{~b}$ ) as $Q$ is effectively constant. Uncertainties, as indicated by the $95 \%$ credible intervals, change drastically prior to around 40-50 Ma where the PPD spans the entire prior range. This indicates that the TI data effectively do not constrain $Q$ before 40-50 Ma. The posterior marginal distribution of $Q_{\operatorname{tmax}}$ (Figure 3d) shows a similar behavior with the expected heat flow approaching the average of the prior, confirming that the data provide progressively less information on $Q$ going further back in time. Between $30 \mathrm{Ma}$ and present day, our algorithm is able to recover the true model well and uncertainties on the retrieved $Q$ model are relatively small, but asymmetric. In particular, between 30 and $5 \mathrm{Ma}$ the upper $95 \%$ credible interval bound indicates that the data constrain the maximum value of $Q$.

The lower credible interval value suggests that $Q$ can be relatively low between 10 and $20 \mathrm{Ma}$, but with low probability (blue color). As a tradeoff, those models tend to have a slightly higher $Q$ between 30 and $50 \mathrm{Ma}$ (just prior to the time of maximum temperature) shown by a trend of lighter blue up to about $50 \mathrm{~mW} \mathrm{~m}^{-2}$ at $50 \mathrm{Ma}$. Given our probabilistic results a more formal correlation analysis of the heat flow parameters is possible in this case (Figure 4). The true value of $Q$ is well recovered but a trend of negative correlation (white dashed line) is observed between heat flow points at 40 and $15 \mathrm{Ma}$, albeit in a region of low probability.

At the present day, the maximum of the posterior distribution of $Q_{\text {pres }}$ is centered on the true value of $40 \mathrm{~mW} / \mathrm{m}^{2}$ (Figure $3 \mathrm{~d}$ ) but slightly skewed toward high values. This effect is due to models that contain rapid variations in heat flow at recent times for which the transient effect does not have enough time to affect the predicted present day temperatures significantly. Because of this effect, the likelihood effectively does not change and so the corresponding models are accepted along the chains.

The histogram of the number of $Q$ time points (Figure 3d) indicates that the minimum number of nodes allowed (one) is generally enough to explain the data. This is expected given the simple (constant) $Q$ model used in the test. However, more complex models (with up to 12 nodes) are still sampled by the algorithm and contribute to the shape of the PPD but with lower probability. The natural parsimony of the algorithm guarantees that, according to the information contained in the data, overly complex $Q$ models (e.g., with more than 12 nodes) are heavily penalized and have zero probability.

The thermal history controls the data fit for TI calibration data. To demonstrate the solutions (including uncertainties) for individual stratigraphic horizons, we show the t-T (time-temperature) curves corresponding to two AFT samples selected over the depth range of the well and their corresponding predicted ages and track length distributions in Figure 5. The results are show that the thermal histories are well resolved at the time of maximum temperature, while thermal histories before that time are generally much less well resolved (except at the time of deposition, which is specified as input).

\subsection{Erosion Event Parameters and the Role of Porosity Data}

As already mentioned, we performed a second inversion, Synthetic Test 2, using the same data set described above except the porosity data. The results for the erosion parameters of both runs are shown in Figure 6a, while the equivalent results presented above for Synthetic Test 1 are given in the SI for the second test (Figure S4). The main contribution of porosity data is to improve the estimate of erosion magnitude associated with a given event. When porosity data are included in the inversion the distribution for $\Delta E$ is tighter, centered on the true value of $2000 \mathrm{~m}$ and with a mean value of 2,027 $\mathrm{m}(S D=56 \mathrm{~m})$. For the inversion without porosity data, the only constraints on $\Delta E$ are the TI data. In this case, the distribution for $\Delta E$ is broader, 
(a)
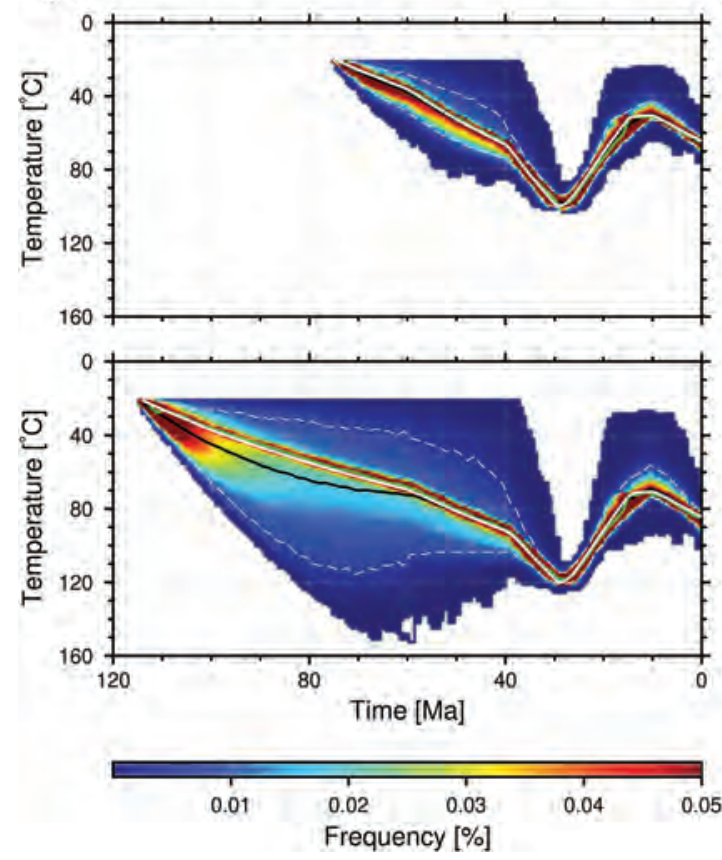

(b)
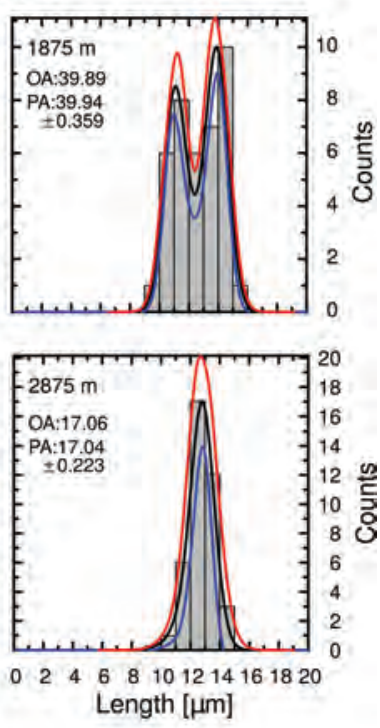

Figure 5. Results of the Synthetic Test 1. (a) Posterior distribution of temperature histories for two selected AFT samples. Black and green lines are the posterior mean and mode with white dashed lines indicating the 95\% credible intervals. White solid lines correspond to the true temperature histories (see Figure 3a). (b) Corresponding AFT length distributions. Histograms represent the observed distributions. Black line is the posterior mean with two standard deviations (red and blue lines). Depth of the samples is indicated in each plot together with the observed (OA) and predicted (PA) AFT ages. For PA, the mean posterior and \pm 1 SD is reported.

positively skewed and with a mean value of 2,229 $\mathrm{m}(S D=226 \mathrm{~m})$. As for the timing parameters, both $t_{\text {start }}$ and $t_{\text {end }}$ are very similar in the two inversions. This indicates that the main constraints for these two time parameters come from the TI data, as we might expect, given the AFT data have explicit time information in the age measurement.

Both variations in $\Delta E$ and $Q$ will affect the predicted temperatures. Intuitively, this should result in a trade-off between these two parameters such that an increase in erosion magnitude will tend to be compensated by a decrease in $Q$ at the time of maximum burial and vice versa. Indeed, in both inversions, this trade-off is clearly observed (Figures $6 \mathrm{~d}$ and $6 \mathrm{e}$ ). However, the information brought by porosity data helps to better constrain $\Delta E$ and in turn, to reduce the ambiguity between erosion and variations in heat flow.

In the two runs described above, the compaction parameters and $T_{s}(t)$ were kept fixed to their true values to reduce possible sources of uncertainties. As a final test with these synthetic data, we inverted the same data set with and without porosity data again but allowed these intermediate parameters to vary. The posterior distributions for these intermediate parameters are given in Figure 7. It is clear that the use of porosity data improves the estimates of compaction parameters (Figures 7a and 7b) although these estimates are strongly correlated (Figure 7e). In contrast, when porosity data are not included in the inversion, the distributions for $\Phi_{0}$ and $c$ are equal to their Gaussian prior distributions and no correlation is observed (Figure 7d). This demonstrates that the TI data provide no constraints on the compaction parameters, although predicted temperatures are conditional on porosity dependent parameters (e.g., thermal conductivity). As for $T_{S}(t)$, its distribution is the same in both cases (Figure 7c), indicating that inference on this parameter is exclusively controlled by TI data, as we would expect. Allowing for uncertain compaction parameters has no effect on the inferred erosion parameters when porosity data are not considered but slightly affects the resolution on $\Delta E$ when porosity data are used (Figure 7f). In more detail, the posterior mean for $\Delta E$ is $2,124 \mathrm{~m}$ $(S D=137 \mathrm{~m})$, which is higher than estimated previously when not perturbing the compaction parameters (which were kept fixed to their true values). This decrease in resolution will be more evident as the 

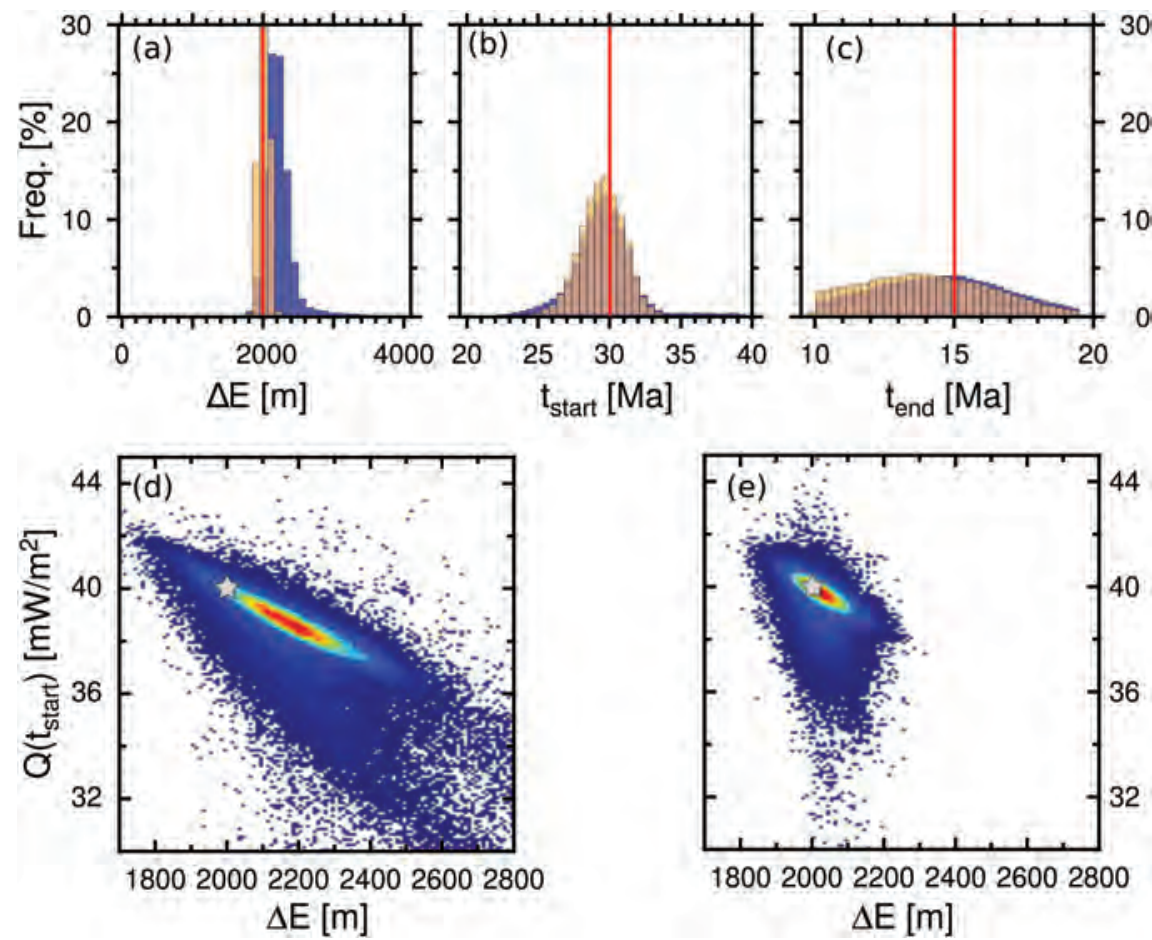

Figure 6. Estimation of erosion-related parameters for Synthetic Tests 1 and 2, when compaction parameters are fixed to the true values. $(\mathrm{a}-\mathrm{c})$ Posterior distribution of the erosion parameters. Blue and yellow histograms refer to the two different inversions performed without and with the porosity data respectively. Red lines indicates the true values. (d) and (e) The posterior 2-D marginal for $\Delta E$ and $Q\left(t_{\text {start }}\right)$ without using porosity data and including them, respectively. Gray stars indicate the true values. $Q\left(t_{\text {start }}\right)$ is approximated by the value of $\mathrm{Q}$ closest to $t_{\text {start }}$ in the interpolated $Q(t)$ for each model. The red to blue colors in (d) and (e) indicate higher to lower probability respectively.

porosity data will contain less information about the compaction parameters and as the prior distributions on compaction parameters get larger. This would allow the algorithm to fit the porosity data with a wider range of $\Delta E$ values. At the same time, the uncertainties on compaction parameters and $T_{s}(t)$ are also mapped on the marginal distribution for $Q$, contributing to increase the overall uncertainties when porosity data are not included (Figure S5).

The results of these synthetic tests demonstrate that the algorithm behaves as expected, showing we can recover parameter values to varying degrees of precision, depending on the nature of the data available and the imposed prior information.

\section{Application to Real Data}

Here we apply our algorithm to a data set provided by Equinor from an unidentified well in the Barents Sea. This specific location was chosen based on the good quality of available data and in particular of porosity data. In addition, estimates of net erosion in the area are available from previous studies (e.g., Ktenas et al., 2018; Licciardi et al., 2019) and will be compared with our results. The TI data consist of four AFT (each with a range of compositional groups, based on $\mathrm{Cl}$ content) and ten VR samples. Two corrected BHT measurements were also available and included in the inversion. A simplified stratigraphy of the borehole is reported in the SI (Table S2). We identify three main lithologies from the shale content $\left(V_{s}\right)$ of the borehole lithostratigraphy, corresponding to sandstone $\left(V_{s h}<10.0 \%\right)$, siltstones $\left(45.0 \%<V_{s h}<55 \%\right)$, and shales $\left(V_{s h}>90.0 \%\right)$, and we compute the proportion of each lithology in each layer (Table S2). A set of representative porosity data was computed by averaging values of the available porosity well-log every $50 \mathrm{~m}$ for each lithology, and we used the standard deviations as the uncertainties for these estimates. 

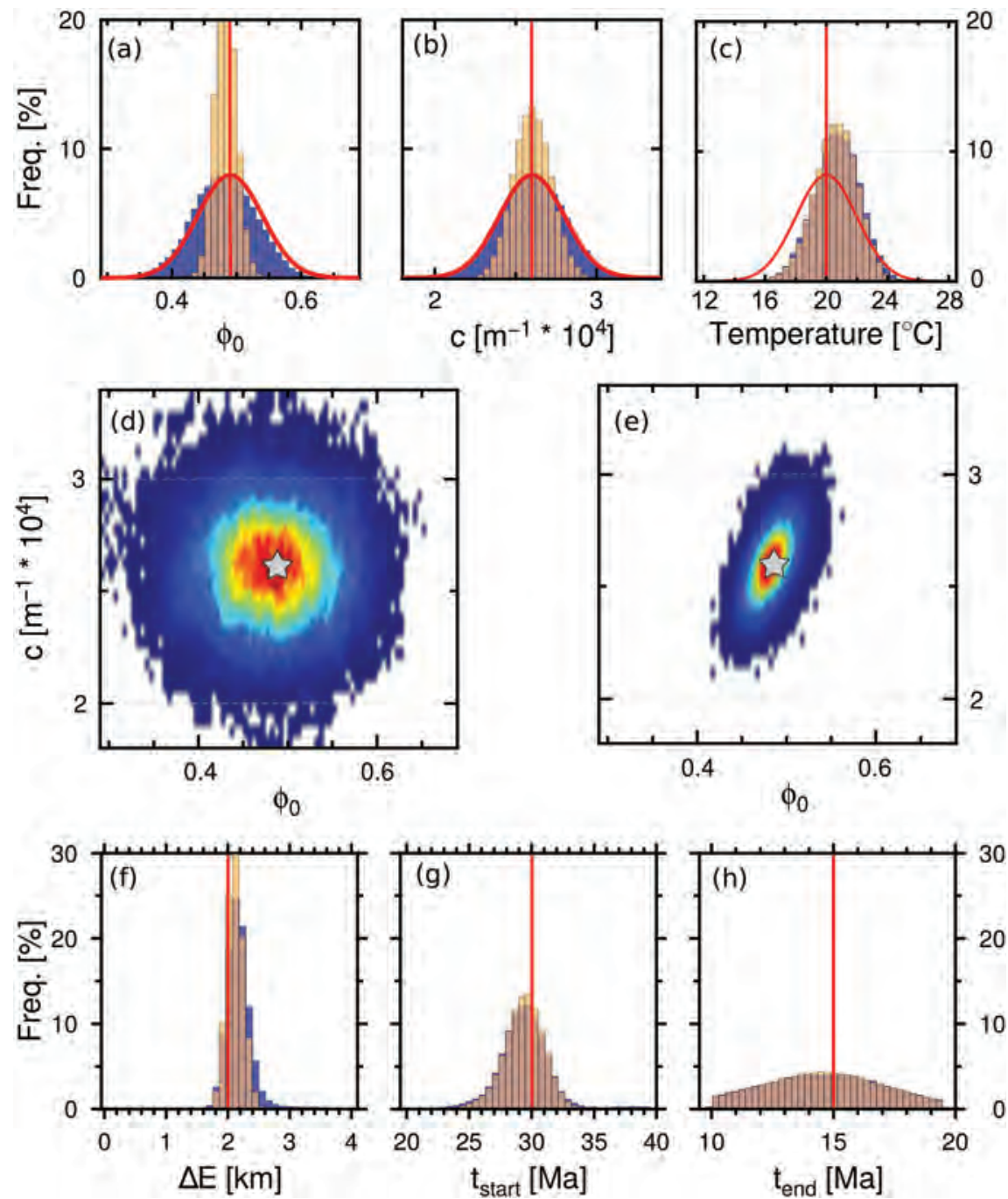

Figure 7. Estimation of intermediate parameters (compaction and surface temperature) for Synthetic Tests 1 and 2. (a-c) Posterior distribution of the intermediate parameters. Blue and yellow histograms refer to the two inversions performed without and with porosity data respectively. Red curves represent the prior distributions centered on the true values (red vertical lines). ( $\mathrm{d}$ and e) The posterior 2-D marginal for the compaction parameters without using porosity data and including them, respectively, and the red to blue colors indicate higher to lower probability respectively. Gray stars indicate the true values. The posterior distribution of the erosion parameters are shown in (f), (g), and (h). Blue and yellow histograms refer to inversions performed without and with porosity data respectively. Red lines indicate the true values.

Prior distributions are reported in Table 1. The compaction parameters for sandstone and siltstone are poorly known in this area, so we chose broad Gaussian prior distributions with mean values equal to those reported in (Sclater \& Christie, 1980). For shale, we use the values obtained by Licciardi et al. (2019) by fitting porosity data from a well in the Barents Sea considered to have not undergone erosion. Values of thermal conductivity (Table S2) were loosely based on previous studies (Gac et al., 2018; Klitzke et al., 2016) and to reflect their dependence on quartz content. However, as discussed in section 2.3 the actual values of thermal conductivity affect the absolute values of heat flow history and not its overall form (Gallagher, 2002). Finally, $T_{s}(t)$ is kept fixed in this example and we used a constant value of $20^{\circ} \mathrm{C}$ from 250 to $30 \mathrm{Ma}$, which decreased linearly down to $4{ }^{\circ} \mathrm{C}$ at the present day.

We perform two different inversions for this data set, similar to the strategy used for the synthetic data. In this case, the first inversion considers only TI and BHT data, while the second also includes porosity data. 
(a)

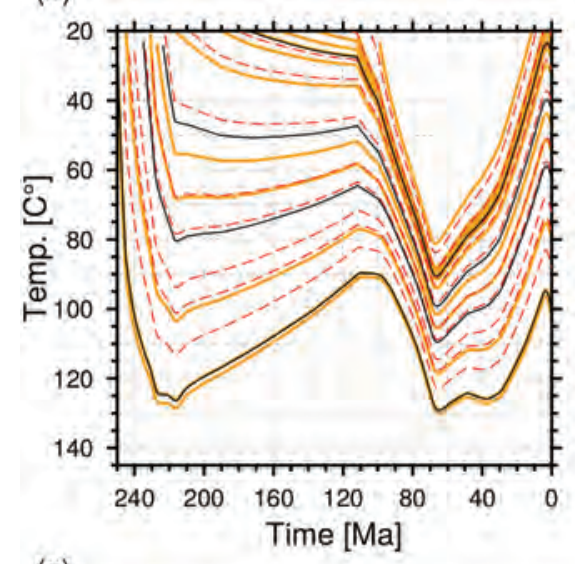

(c)

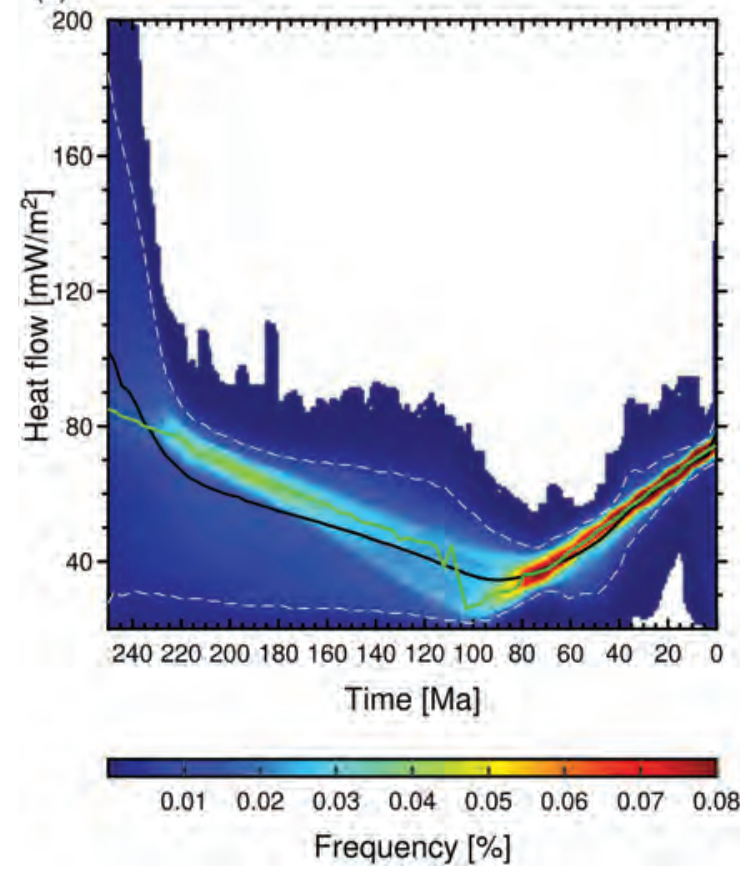

(b)

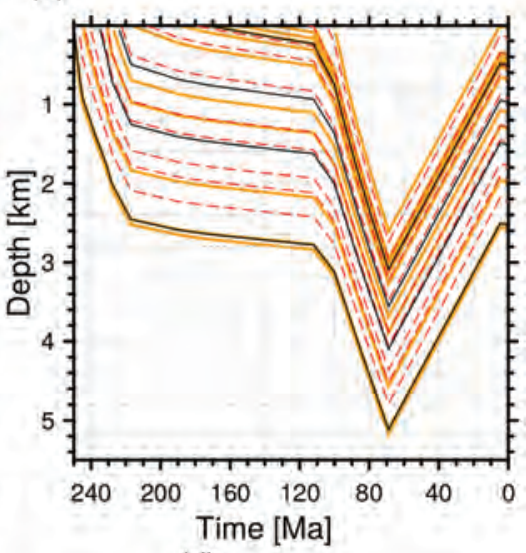

(d)

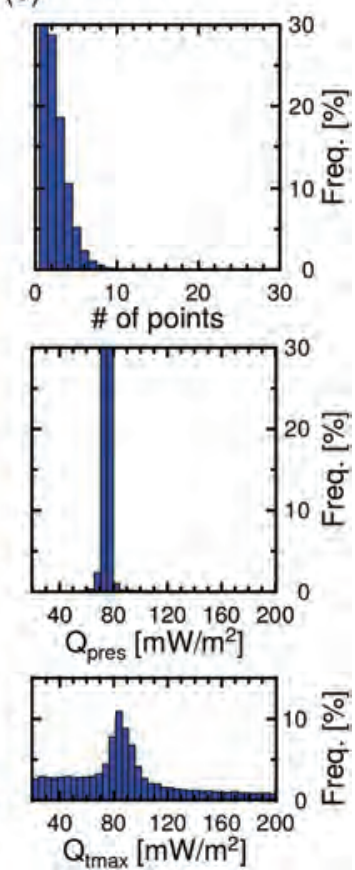

Figure 8. Results of inversion of real data using VR, AFT, BHT, and porosity data. (a) Temperature histories and (b) burial histories. These are calculated using the posterior mean heat flow model (black line in c) and erosion parameters. Stratigraphic layers are indicated by orange lines, VR samples with dashed red lines, and AFT samples with black lines. (c) Marginal posterior distributions of $Q(t)$. The black and green lines show the posterior estimates of the mean and mode $Q$ models, respectively. White dashed lines indicate $95 \%$ credible intervals. (d) Marginal posterior distribution for the number of nodes, $Q_{\text {pres }}$ and $Q_{t \max }$.

The posterior results for $Q$ are very similar and so we only show those for the more complete data set in Figure 8, while those for the reduced data set are given in the SI (Figure S6). The best constrained part of the model is between $80 \mathrm{Ma}$ and the present day, for which a linear increase of heat flow from about 40 to about $75 \mathrm{~mW} \mathrm{~m}^{-2}$ is inferred. Before that, only the upper bound of $Q$ is constrained (with the upper $95 \%$ credible interval in the $70-80 \mathrm{~mW} \mathrm{~m}^{-2}$ range between 220 and $100 \mathrm{Ma}$ ). Between 250 and $220 \mathrm{Ma}$ the marginal posterior distribution spans almost the entire prior range, indicating that the data contain little information in this time window.

In more detail, the distribution of $Q$ from Figure $8 \mathrm{c}$ implies bimodality. This bimodality is clearly seen as a jump in the mode $Q$ model (the green line in Figure 8c). The mode model is constructed by connecting the heat flow value with highest marginal probability at successive times (1 Myr intervals). The distribution of 
(a)

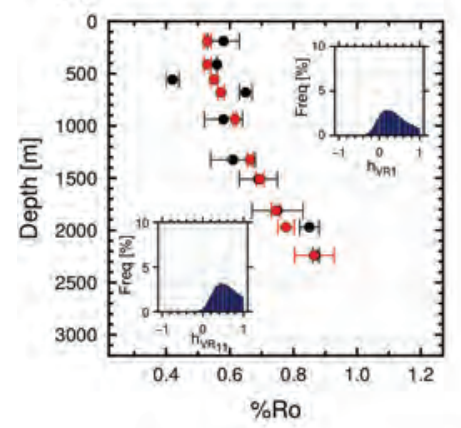

(d) (b)

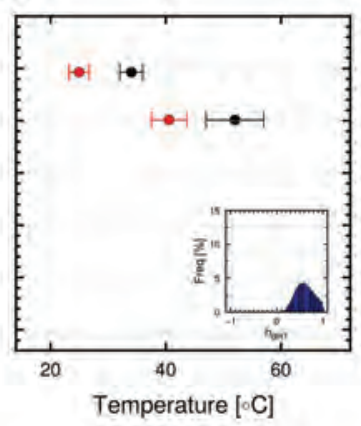

(c)

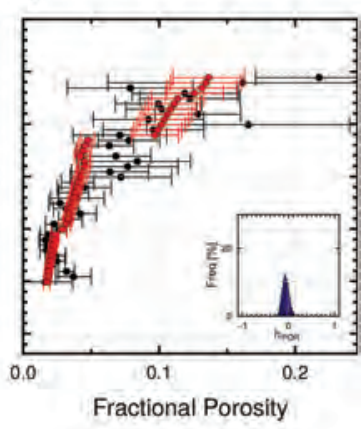

(e)
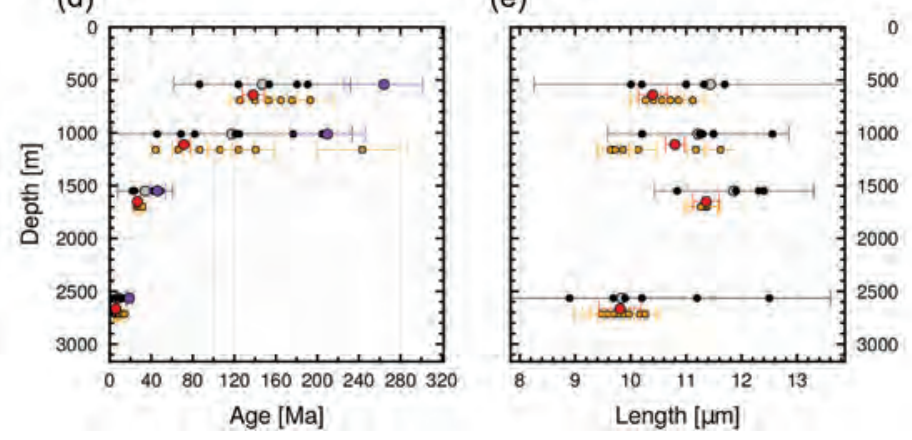

Figure 9. Data fit for the real data example discussed in the main text. Observed data are indicated with black circles and error bars are \pm 1 standard deviation. The error bars on the predicted values (red circles) represent the \pm 2 standard deviation range (the 95\% credible range for a Gaussian distribution) for the predictions from all models during the inversion. The insets on the top three panels show the distributions of the weighting parameter $\left(h_{i}\right)$ for the relevant data value or data set (the input errors are weighted by $10^{h_{i}}$ so that $h_{i}<0$ leads to weights $<1$ ). (a) VR (here we show just the weighting parameter for the shallowest and deepest data values), (b) BHT, (c) porosity, (d) AFT age, and (e) AFT mean track length. Note for the AFT data the predicted values are offset in depth for clarity, and the range of values represents different compositional groups ( $\mathrm{Cl}$ content in $0.1 \mathrm{wt} \%$ intervals). Gray circles are obtained by averaging the observed values of each compositional bin (small black circles) for a given sample. Posterior mean predictions (red circles) are computed as a weighted mean of the predictions for each individual bin (yellow circles). In (d), the purple circles to the right of the AFT ages indicate the posterior mean predictions (weighted mean of each individual compositional bin) for the effective closure time, or when the AFT system begins to retain thermal history information, for each sample.

models suggests a tightly constrained decrease in heat flow back to $80 \mathrm{Ma}$, then a divergence into two broad modes, the first of which has increasing heat flow back in time, while the other continues to decrease until about 100-110 Ma. The two modes come together again around $160 \mathrm{Ma}$. The average $Q$ model falls between the two modes as expected with a bimodal distribution. We return to this below.

A visual comparison of the predictions with the observed data for this inversion is given in Figure 9. In general, the main trends in the data are well reproduced, although the AFT observations are more dispersed than the predicted values. Also, the BHT data are somewhat underpredicted. However, the estimated hyperparameter for BHT data (Figure 9b) implies a scaling factor of around 3-4 of the observed errors (Equation 10). The observed data are fit to within the rescaled errors and therefore, they have less importance in the inversion (see section 2.4). In contrast, the algorithm attributes more importance to the porosity data by reducing their input errors (the hyperparameter is $<0$ in Figure 9c). As for VR data points, their hyperparameters show higher variability. This is due to the fact that having one hyperparameter for each data point allows for a wider range of solutions that fit the data equally well. Here, we note that we tested alternative VR kinetic parameters to Easy $\% R_{o}$ (i.e., Basin $\% R_{o}$; Nielsen et al., 2017). The results are reported in the SI (Figures S7 and S8) for the interested reader, as a full comparison between different VR models goes beyond the scope of this work.

As seen for the synthetic data case, the benefit of including porosity data is evident when estimating $\Delta E$ (Figure 10a). In this case, the distribution for $\Delta E$ is relatively symmetric, narrower and shifted to slightly 
(a)

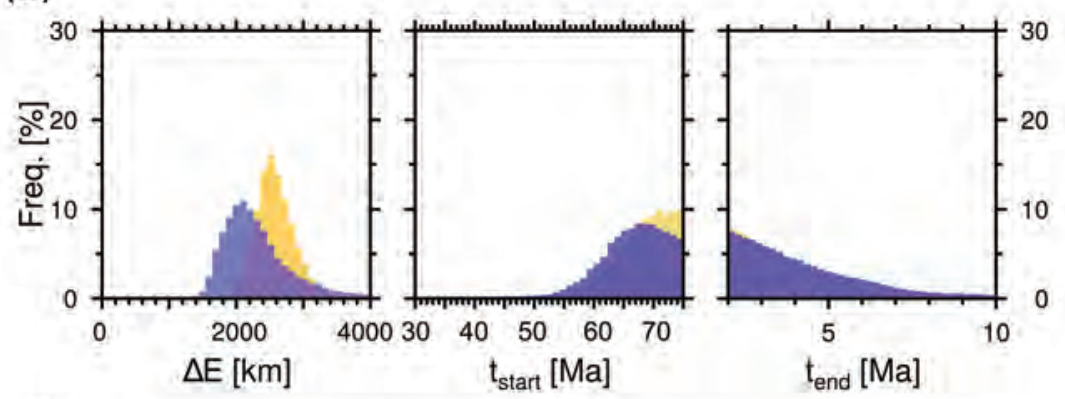

(b)

(c)

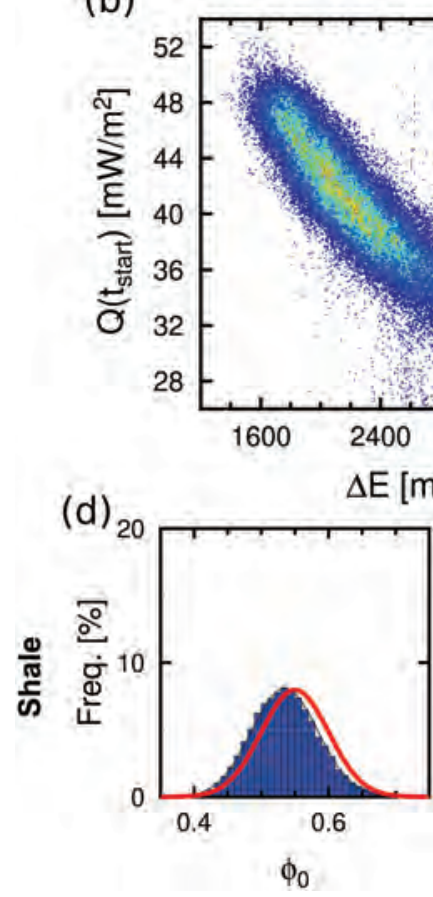

Figure 10. Results of the inversion of real data. (a) Posterior distribution of $\Delta E, t_{\text {start }}$ and $t_{\text {end }}$. Blue and yellow histograms refer to inversions without and with porosity data. In both cases VR, AFT, and BHT are also used.

(b and c) The posterior 2-D marginal for $\Delta E$ and $Q\left(t_{\text {start }}\right)$ without using porosity data and including them, respectively. $Q\left(t_{\text {start }}\right)$ is approximated by the value of $Q$ closest to $t_{\text {start }}$ in the interpolated $Q(t)$ for each model. (d) Posterior distributions for the compaction parameters, $\Phi_{0}$ and $c$, for shale, the right side panel shows their joint distribution, blue to red implies higher probability. Red curves indicate the Gaussian prior distributions. The results for sandstone and siltstone are shown in Figure S9 of the SI.

higher values than that obtained with TI data only, which in contrast is positively skewed. Negligible differences are observed for $t_{\text {end }}$, while the distribution for $t_{\text {start }}$ implies a preference for a slightly older value when the porosity data are included. Although the main control on the timing of erosional event is provided by TI data, the observed differences are due to the trade-off between $Q$ and the maximum burial depth (i.e., the burial history), which are adjusted in the inversion according to the porosity data fit. As observed for synthetic data, the trade-off between $\Delta E$ and $Q$ is reduced when porosity data are considered (Figures 10b and 10c).

The compaction parameters predominantly control the fit of the porosity data and in turn, determine the PPD of $\Delta E$. In Figure 10d we also show the PPDs for $\Phi_{0}$ and $c$ relative to shale (the same results for sandstone and siltstone are shown in Figure S9 of the SI). Shale represents the dominant lithology in the most robust portion (with smaller uncertainties) of the porosity log data (Figure 9c) between 1,137 and 2,560 m (Table $\mathrm{S} 2$ ). Our results indicate that higher values of $c$ than the assumed prior mean, and so a higher compaction 
(a)
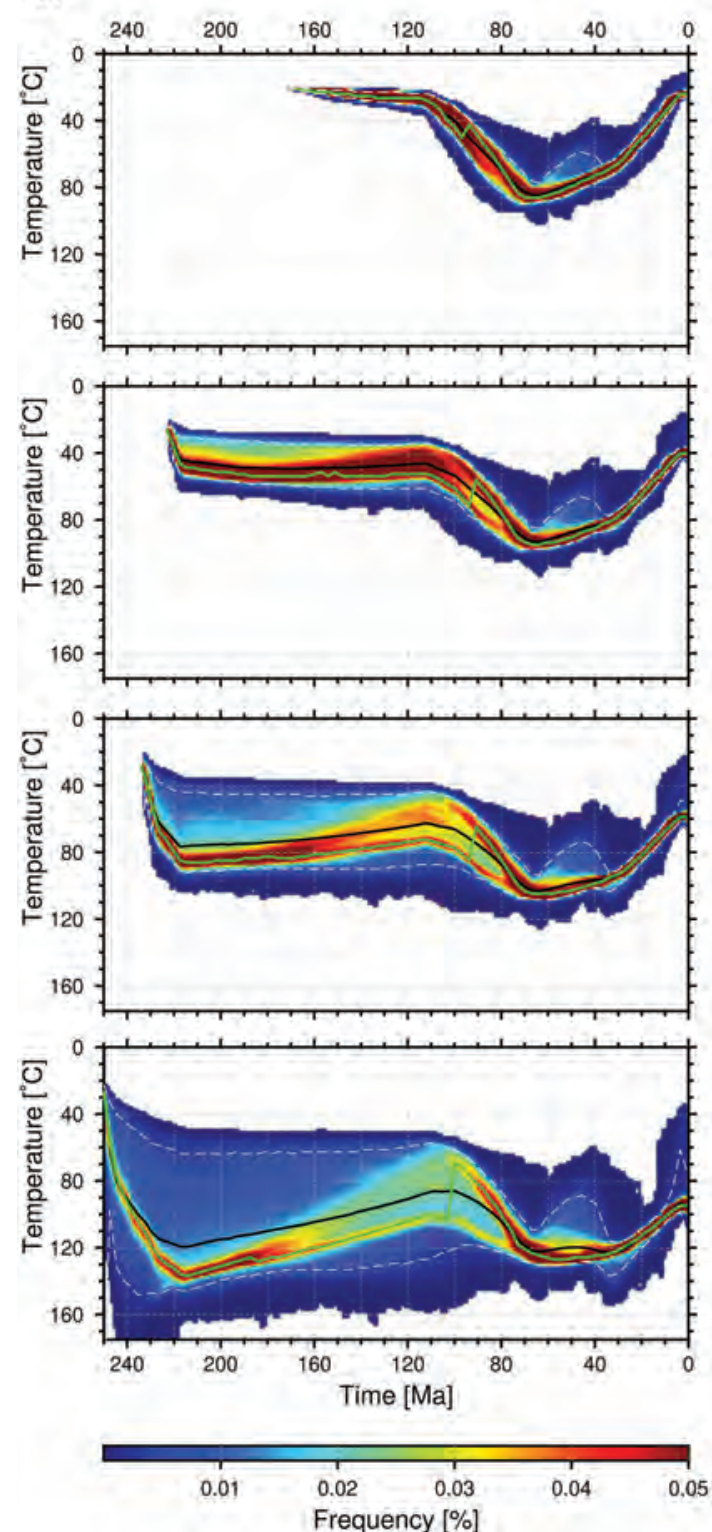

(b)
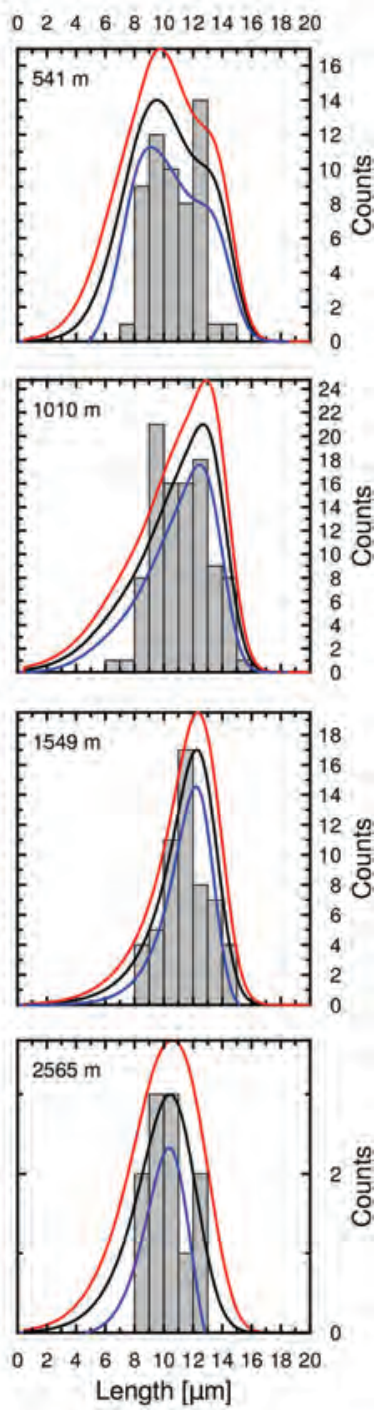

Figure 11. Results of the inversion of real data. (a) Posterior distribution of temperature histories for the four AFT samples. Black and green lines indicate posterior mean and mode respectively. White dashed lines are the $95 \%$ credible intervals. (b) Corresponding length distributions. Histograms represent the observed distributions. Black line is the posterior mean with two standard deviations (red and blue lines). Depth of the samples is indicated in each plot. See Figure $9 \mathrm{~d}$,e for a comparison of the predicted and observed ages and mean track lengths.

rate as a function of depth, are required in order to fit the porosity data. The joint distribution of the two compaction parameters also implies a slight positive correlation (see Figure 10b).

Lastly, we examine the posterior distribution of time-temperature curves for the four AFT samples (Figure 11). The maximum paleotemperatures are located around the time of maximum burial at the inferred value of $t_{\text {start }}$ (65-75 Ma in Figure 10a), which corresponds to a minimum value in $Q$ of about 40 $\mathrm{mW} \mathrm{m}{ }^{-2}$ (Figure 8 ). However, between 70 to about $30 \mathrm{Ma}$, small variations in temperature occur, with the deepest layer showing an almost constant value $\left(125-130^{\circ} \mathrm{C}\right)$ and the shallowest samples indicating a cooling trend of $20-25^{\circ} \mathrm{C}$. This reflects the decreasing heat flow from around $80 \mathrm{Ma}$ combined with a component of advective heat transfer toward the surface. The latter effect means that as sample depth decreases 
in response to erosion, the sample will tend to be hotter relative to the no advection case. This is more significant for the deeper samples as they are less strongly influenced by the upper boundary condition given by $T_{s}(t)$. After $30 \mathrm{Ma}$, all samples cool relatively continuously, with a minor increase in temperatures after $2 \mathrm{Ma}$.

As mentioned above, the earlier part of the $Q$ model appears to be bimodal (Figure 8). In terms of the temperature histories, the bimodality is more apparent for the deeper samples as a given change in heat flow leads to a greater change in temperature relative to the shallower samples. In particular, the two deeper samples in Figure 11 show a broad spread (the green/yellow colors) in the thermal histories around 110-100 Ma, which is more or less bimodal (light blue in the center). One mode implies cooling from about $140^{\circ} \mathrm{C}$ at 220 Ma to around $100^{\circ} \mathrm{C}$ and the other mode cooling to about $60-70^{\circ} \mathrm{C}$ at $110 \mathrm{Ma}$. The first (higher temperature) mode in the thermal histories reflects the first (higher $Q$ ) mode discussed above, and we see the same discontinuity in the modal thermal history around 110-100 Ma.

It is important to note that earlier parts of the thermal histories are not constrained by the deeper AFT data nor is the heat flow increase from $80 \mathrm{Ma}$ back to $220 \mathrm{Ma}$. The deeper samples just need to be hot enough and then cool below the effective closure temperature $\left(110 \pm 10^{\circ} \mathrm{C}\right.$ typically) recently enough to explain the very young AFT ages. This bimodality is attributable to the deeper VR data, which can be reasonably predicted either by high temperatures around 220 or $70 \mathrm{Ma}$ (see Figure 8a). The latter event (70 Ma) is important for predicting the AFT data, which are not sensitive to the earlier maximum temperatures. High heat flow is needed to get high temperatures at $220 \mathrm{Ma}$, as the maximum burial depth is around 2,500 m. At $70 \mathrm{Ma}$, the same temperature is obtained with a lower heat flow as the burial depth is around 5,000 $\mathrm{m}$.

Similar to what we observed in the synthetic test (Figure 4), an implied trade-off can be seen in the heat flow histories as bumps in the credible intervals between 60 and $30 \mathrm{Ma}$. Prior to $40 \mathrm{Ma}$, the heat flow can decrease relative to the modal/mean value and then increase to be slightly higher than the modal/mean heat flow between 40 and $30 \mathrm{Ma}$. These ambiguities (bimodal solutions and oscillations) demonstrate that the data contain different information or constrain the heat flow history differently. Some of this ambiguity could be eliminated with different and tighter prior ranges on the heat flow values, or even rates of change of heat flow, but that is beyond the scope of this work.

\section{Discussion}

Given that TI data are a strong function of the maximum temperature, it is intuitive that TI data provide constraints on $Q$ primarily at the time of maximum paleotemperature and the evolution of $Q$ after that time. The ability to resolve more detail will depend on the range of timing of maximum temperature for samples at different depths, the nature of the data available, and the information contained in the data about different parts of the $Q$ model. However, our probabilistic inversion makes it easier to assess both the well and less well resolved portions of the model through quantification of the posterior uncertainties.

Our results show that TI data can be used alone to quantify heat flow and erosion histories and their uncertainties. In general, some form of trade-off between $\Delta E$ and $Q$ is to be expected, again reflecting the significance of the maximum temperatures, but such trade-offs should be evident in the joint distributions of heat flow and erosion magnitude. Porosity data can also be used to provide additional and temperature independent constraints on $\Delta E$ which, conditional on the data errors, can reduce the range of possible solutions. Our simple synthetic tests show that some of the erosional parameters can be difficult to constrain $\left(t_{\text {end }}\right.$ in Figure $6 \mathrm{c}$ ). For example, $t_{\text {start }}$ and $t_{\text {end }}$ are pretty much exclusively constrained by TI data and in particular by AFT data, which contain implicit timing information in the measured ages. The lack of information about erosion parameters can easily introduce complex trade-offs between other model parameters. Often, we may be more interested in quantifying $\Delta E$ given an assumed duration and age of an erosional event. In this case, tighter priors on $t_{\text {start }}$ and $t_{\text {end }}$ should be used. As presented, our choice of parametrization for an erosional event may be considered simplistic as we do not allow for multi-stage or variable rate events. While it is debatable as to whether the calibration data can resolve a more complex history in a single erosion event, our choice here should be considered as a proof of concept, and is open to further developments.

Concerning the real data case, it was not the aim of this work to provide a comprehensive interpretation of thermal evolution of the Barents Sea. Indeed this is not possible from a single well. However, our estimates of 
$\Delta E$ are in agreement with previous studies that reported between 1.8 and $2.5 \mathrm{~km}$ of net erosion for the same area (Ktenas et al., 2018; Licciardi et al., 2019). Moreover, including porosity data in the analysis contributes to reduce the uncertainties associated with $\Delta E$ (see Figure 10a). More importantly, our approach has the potential to reconcile TI- and compaction-derived estimates of net erosion, which can be difficult, often resulting in divergent estimates (e.g., Burns et al., 2005). Furthermore, this example demonstrated the role of different data in determining the distributions of acceptable solutions. We found bimodality and some minor trade-off in parts of the heat flow histories, the bimodality being attributable to the VR data. Strategies to assess the sensitivity of the solutions to different data types in the inferred solutions will be case-specific and problem dependent. In general, however, this aspect can partially be addressed by running inversions with just one data type, or different combinations of data types, while still making predictions of the excluded calibration data as a control.

In addition to heat flow and erosion histories, the posterior distributions of temperature histories are readily available, as is often desired in the context of hydrocarbon exploration (Hantschel \& Kauerauf, 2009). Temperature histories are determined by both the $Q$ history and variations of the burial histories which may be considered as partially unknown (through the erosion parameters). This allows us to use imprecise stratigraphic data as geological constraints in the inversion, and the uncertainties on erosional events are readily propagated through to the temperature histories. The algorithm also deals with the possibility of pre-depositional thermal history component although this feature has not been demonstrated here. This is important for AFT data when they can contain inherited or provenance related fission tracks if the postdepositional thermal history does not lead to total annealing. We parametrize this by adding single pre-depositional time-temperature point to the thermal history for each AFT sample (as in Figure 10 of Gallagher, 2012).

As mentioned in section 1, additional data types used in basin modeling can be easily incorporated into our algorithm, provided the appropriate predictive models are available and that a likelihood function can be defined. Additionally, here the algorithm has been applied to data from a single borehole, but further developments should involve the use of multiple boreholes in a joint inversion to constrain the time-evolution of $Q$ in a given region. This may be done by some kind of spatial clustering/classification approach as has been previously proposed for thermochronological data (Stephenson et al., 2006) and borehole temperature data used in climate reconstructions (Hopcroft et al., 2009).

As for the intermediate parameters, here we have considered $T_{s}(t), \Phi_{0}$ and $c$. In principle, other parameters could be considered in the same way, for example, reaction kinetics, thermal conductivity, heat production, and stratigraphic ages of the sediments. Although this would be an effective way of mapping their uncertainties onto the posterior uncertainties of $Q$, increasing the number of the intermediate parameters could cause their uncertainties to overwhelm the information contained in the data, resulting in poorly constrained solutions. A more useful way of dealing with uncertain intermediate parameters would be to adopt a Bayesian model choice approach (e.g., Friel \& Wyse, 2012). This allows a quantitative comparison between inversions with different choices of the parameters, thus helping the user to identify the relative importance of different parameters and to make a quantitative assessment of competing or alternative model parametrizations.

\section{Conclusion}

We have presented a new approach for thermal history reconstruction in basin modeling. The newly developed rjMcMC algorithm makes use of transdimensional Bayesian inference to reconstruct the heat flow history of a sedimentary basin given multiple data types and stratigraphic information collected from a single borehole. The advantages brought by our fully nonlinear stochastic approach over previous inversion approaches are general and can be summarized as follows: (i) There is no dependence on the starting model, (ii) we use adaptive parametrization (i.e., complexity of the heat flow history is dictated solely by the data), (iii) quantification of formal uncertainties (including correlation) on model parameters is straightforward, and (iv) we can allow for variable or unknown data errors.

In the context of basin modeling, we have shown that the algorithm can be applied reliably to quantify the uncertainties on the retrieved $Q$ model given prior information and assumptions on the forward problem and data error statistics. We have presented the first attempt (to the authors knowledge) to probabilistically quantify the effect of erosional events on heat flow history reconstruction, which is key in basin modeling. In 


\section{Acknowledgments}

The authors would like to thank Andrea Stevens Goddard and Matthew Fox for their suggestions and comments, which helped to strongly improve the quality of the manuscript. A. L. is indebted to Nicola Piana Agostinetti for sharing his rjMcMC algorithm, from which A. L. took inspiration. The authors also thank Malcom Sambridge for making his Parallel Tempering routines available online. addition, we have shown how uncertainties on "intermediate" parameters, such has surface temperatures and compaction parameters can be effectively propagated to the final model by sampling along the transdimensional chains. Although in this work we used relatively broad prior distributions, our Bayesian approach allows for straightforward implementation of tighter prior distributions, that is "geological constraints" for all parameters.

\section{Data Availability Statement}

The synthetic data used in this work can be downloaded from https://doi.org/10.6084/m9.figshare.12459071. v1 website. A request to obtain the data files used for the real data test case can be submitted to K. G., conditional on obtaining permission from Equinor.

\section{References}

Allen, P. A., \& Allen, J. R. (2005). Basin analysis. Malden, MA: Blackwell Publishing.

Bodin, T., Salmon, M., Kennett, B. L. N., \& Sambridge, M. (2012). Probabilistic surface reconstruction from multiple data sets: An example for the Australian Moho. Journal of Geophysical Research, 117, B10307. https://doi.org/10.1029/2012JB009547

Bodin, T., Sambridge, M., Tkalčić, H., Gallagher, K., Arroucau, P., \& Rawlinson, N. (2012). Transdimensional inversion of receiver functions and surface wave dispersion. Journal of Geophysical Research, 117, B02301. https://doi.org/10.1029/2011JB008560

Burns, W. M., Hayba, D. O., Rowan, E. L., \& Houseknecht, D. W. (2005). Estimating the amount of eroded section in a partially exhumed basin from geophysical well logs: An example from the north slope. U.S. Geological Survey Professional Paper, 1732-D, 1-18.

Charvin, K., Gallagher, K., Hampson, G. L., \& Labourdette, R. (2009). A Bayesian approach to inverse modelling of stratigraphy, Part 1: Method. Basin Research, 21, 5-25.

Corcoran, D. V., \& Doré, A. G. (2005). A review of techniques for the estimation of magnitude and timing of exhumation in offshore basins. Earth-Science Reviews, 72, 129-168.

Dettmer, J., Dosso, S. E., \& Holland, C. W. (2010). Trans-dimensional geoacoustic inversion. Journal of the Acoustical Society of America, $128,3393-3405$.

Dosso, S. E., Dettmer, J., Steininger, G., \& Holland, C. W. (2014). Efficient trans-dimensional Bayesian inversion for geoacoustic profile estimation. Inverse Problem, 30, 114018. https://doi.org/10.1088/0266-5611/30/11/114018

Falcioni, M., \& Deem, M. W. (1999). A biased Monte Carlo scheme for zeolite structure solution. The Journal of Chemical Physics, 110, 1754-1766.

Ferrero, C., \& Gallagher, K. (2002). Stochastic thermal history modelling. 1. Constraining heat flow histories and their uncertainty. Marine and Petroleum Geology, 19, 633-648.

Friel, N., \& Wyse, J. (2012). Estimating the evidence-A review. Statistica Neerlandica, 66(3), 288-308. https://doi.org/10.1111/j.14679574.2011.00515.X

Gac, S., Hansford, P. A., \& Faleide, J. I. (2018). Basin modelling of the SW Barents Sea. Marine and Petroleum Geology, 95, $167-187$.

Gallagher, K. (1989). An examination of some uncertainties associated with estimates of sedimentation rates and tectonic subsidence. Basin Research, 2, 97-114.

Gallagher, K. (1995). Evolving temperature histories from apatite fission-track data. Earth and Planetary Science Letters, 136, 421-435. https://doi.org/10.1016/0012-821X(95)00197-K

Gallagher, K. (1998). Inverse thermal history modelling as a hydrocarbon exploration tool. Inverse Problems, $14,479-497$.

Gallagher, K. (2002). Modelling thermal histories-How important are thermal conductivity data? In R. Marzi, \& S. Düppenbecker (Eds.), Multidimensional basin modelling (Vol.7, pp. 1-9). Tulsa, OK: AAPG/Datapages Discovery Series.

Gallagher, K. (2012). Transdimensional inverse thermal history modeling for quantitative thermochronology. Journal of Geophysical Research, 117, B02408. https://doi.org/10.1029/2011JB008825

Gallagher, K., Brown, R. W., \& Johnson, C. J. (1998). Geological applications of fission track analysis. Annual Reviews of Earth and Planetary Sciences, 26, 519-572.

Gallagher, K., Charvin, K., Nielsen, S., Sambridge, M., \& Stephenson, J. (2009). Markov chain Monte Carlo (MCMC) sampling methods to determine optimal models, model resolution and model choice for earth science problems. Marine and Petroleum Geology, 26, 525-535.

Gallagher, K., \& Sambridge, M. (1992). The resolution of past heat flow in sedimentary basins from non-linear inversion of geochemical data: The smoothest model approach, with synthetic examples. Geophysical Journal International, 109, 78-95.

Geyer, C. J. (1991). Markov Chain Monte Carlo maximum likelihood, in Computing Science and Statistics: Proceedings of the 23rd Symposium on the Interface. American Statistical Association, 156-163.

Green, P. J. (1995). Reversible jump MCMC computation and Bayesian model selection. Biometrika, 82, 711-732.

Green, P. J. (2003). Trans-dimensional Markov chain Monte Carlo. Highly Structured Stochastic Systems, 27, 179-198.

Green, P. F., \& Duddy, I. R. (2012). Thermal history reconstruction in sedimentary basins using apatite fission-track analysis and related techniques, Analyzing the thermal history of sedimentary basins: Methods and case studies (Vol.103, pp. 65-104). Tulsa, OK: SEPM Special Publication.

Hantschel, T., \& Kauerauf, A. (2009). Fundamentals of basin and petroleum systems modeling. Berlin Heidelberg: Springer-Verlag.

Hastings, W. K. (1970). Monte Carlo sampling methods using Markov chains and their applications. Biometrica, $57,97$.

Hopcroft, P., Gallagher, K., \& Pain, C. C. (2009). A Bayesian partition modelling approach to resolve spatial variability in climate records from borehole temperature inversion. Geophysical Journal International, 178, 651-666. https://doi.org/10.1111/j.1365-246X.2009.04192

Jasra, A., Stephens, D. A., Gallagher, K., \& Holmes, C. C. (2006). Bayesian mixture modelling in geochronology via Markov chain Monte Carlo. Mathematical Geosciences, 38, 269-300.

Ketcham, R. A. (2005). Forward and inverse modeling of low-temperature thermochronometry data. Reviews in Mineralogy \& Geochemistry, $58,275-314$.

Ketcham, R. A., Carter, A., Donelick, R. A., Barbarand, J., \& Hurford, A. J. (2007). Improved modeling of fission-track annealing in apatite. American Mineralogist, 92, 799-810. 
Ketcham, R. A., Donelick, R. A., \& Carlson, W. D. (1999). Variability of apatite fission-track annealing kinetics. III. Extrapolation to geological timescales. American Mineralogist, 84, 1235-1255.

Klitzke, P., Sippel, J., Faleide, J. I., \& Scheck-Wenderoth, M. (2016). A 3D gravity and thermal model for the Barents Sea and Kara Sea. Tectonophysics, 684, 131-147.

Ktenas, D., Meisingset, I., Henriksen, E., \& Nielsen, J. K. (2018). Estimation of net apparent erosion in the SW Barents Sea by applying velocity inversion analysis. Petroleum Geoscience.

Lerche, I. (1988). Inversion of multiple thermal indicators: Quantitative methods of determining paleoheat flux and geological parameters I. Theoretical development for paleoheat flux. Mathematical Geology, 20, 1-36.

Licciardi, A., England, R. W., Piana Agostinetti, N., \& Gallagher, K. (2020). Moho depth of the British Isles: A probabilistic perspective. Geophysical Journal International, 221, 1384-1401.

Licciardi, A., Gallagher, K., \& Clark, S. A. (2019). Estimating uncertainties on net erosion from well-log porosity data. Basin Research, $00,1-17$.

Mackay, D. J. C. (2003). Information theory, inference, and learning algorithms. Cambridge: Cambridge University Press.

Malinverno, A. (2002). Parsimonious Bayesian Markov chain Monte Carlo inversion in a nonlinear geophysical problem. Geophysical Journal International, 151, 675-688.

Malinverno, A., \& Briggs, V. (2004). Expanded uncertainty quantification in inverse problems: Hierarchical Bayes and empirical Bayes. Geophysics, 69, 1005-1016.

Malusà, M. G., \& Fitzgerald, P. G. (2018). Fission-Track thermochronology and its application to geology: Springer International Publishing AG

Mandolesi, E., Ogaya, X., Campanyà, J., \& Piana Agostinetti, N. (2018). A reversible-jump Markov chain Monte Carlo algorithm for 1D inversion of magnetotelluric data. Computers and Geosciences, 113, 94-105.

McCulloh, T. H., \& Naeser, N. D. (1989). Thermal history of sedimentary basins: Introduction and overview. In T. H. McCulloh, \& N. D. Naeser (Eds.), Thermal history of sedimentary basins. New York, NY: Springer.

Metropolis, N., Rosenbluth, A. W., Rosenbluth, N. M., Teller, A. H., \& Teller, E. (1953). Equation of state calculations by fast computing machines. The Journal of Chemical Physics, 21(6), 1087-1092.

Mosegaard, K., \& Tarantola, A. (1995). Monte Carlo sampling of solutions to inverse problems. Journal of Geophysical Research, 100(B7), $12,431-12,447$.

Nielsen, S. B. (1996). Sensitivity analysis in thermal and maturity modelling. Marine and Petroleum Geology, 13, 415-425.

Nielsen, S. B., Clausen, O. R., \& McGregor, E. (2017). Basin $\% r_{o}$ : A vitrinite reflectance model derived from basin and laboratory data. Basin Research, 29(Suppl.1), 515-536. https://doi.org/10.1111/bre.12160

Piana Agostinetti, N., \& Bodin, T. (2018). Flexible coupling in joint inversions: A Bayesian structure decoupling algorithm. Journal Geophysical Research: Solid Earth, 123, 8798-8826. https://doi.org/10.1029/2018JB016079

Piana Agostinetti, N., Giacomuzzi, G., \& Malinverno, A. (2015). Local 3D earthquake tomography by trans-dimensional Monte Carlo sampling. Geophysical Journal International, 201, 1598-1617.

Piana Agostinetti, N., \& Malinverno, A. (2010). Receiver function inversion by trans-dimensional Monte Carlo sampling. Geophysical Journal International, 181, 858-872.

Ravenna, M., \& Lebedev, S. (2017). Bayesian inversion of surface-wave data for radial and azimuthal shear-wave anisotropy, with applications to central Mongolia and west-central Italy. Geophysical Journal International, 213(1), 278-300. https://doi.org/10.1093/gji/ggx497

Ray, A., Sekar, A., Hoversten, M., \& Albertin, U. (2016). Frequency domain full waveform elastic inversion of marine seismic data from the Alba field using a Bayesian trans-dimensional algorithm. Geophysical Journal International, 205, 915-937.

Reading, A., \& Gallagher, K. (2013). Transdimensional changepoint modelling as a tool to investigate uncertainty in applied geophysical inference: An example using borehole geophysical logs. Geophysics, 78, 89-99.

Robert, C., \& Casella, G. (2004). Monte Carlo statistical methods. New York: Springer-Verlag.

Sambridge, M. (2013). A Parallel Tempering algorithm for probabilistic sampling and multimodal optimization. Geophysical Journal International, 196, 357-374.

Sambridge, M., Bodin, T., Gallagher, K., \& Tkalčić, H. (2013). Transdimensional inference in the geosciences. Philosophical Transactions of the Royal Society A, 371, 20110547.

Schneider, D., \& Issler, D. (2019). Application of low-temperature thermochronology to hydrocarbon exploration. In M. Malusa, \& P. F. Fitzgerald (Eds.), Fission-track thermochronology and its applications to geology (pp. 315-333). Cham: Springer.

Sclater, J. G., \& Christie, P. A. F. (1980). Continental stretching: An explanation of the post-mid-Cretaceous subsidence of the central North Sea basin. Journal of Geophysical Research, 85, 3711-3739.

Stephenson, J., Gallagher, K., \& Holmes, C. C. (2006). Low temperature thermochronology and strategies for multiple samples 2: Partition modelling for 2D/3D distributions with discontinuites. Earth and Planetary Science Letters, 241, 557-570.

Sweeney, J. J., \& Burnham, A. K. (1990). Evaluation of a simple model of vitrinite reflectance based on chemical kinetics. AAPG Bullettin, $74,59-70$.

Waples, D. W. (1994). Maturity modeling: Thermal indicators, hydrocarbon generation, and oil cracking. The Petroleum System From Source to Trap, American Association of Petroleum Geologists, https://doi.org/10.1306/M60585C17

Waples, D. W., Kamata, H., \& Suizu, M. (1992). The art of maturity modeling, Part 1: Finding a satisfactory geologic model. AAPG Bullettin, $76,31-46$.

Waples, D. W., Kamata, H., \& Suizu, M. (1992). The art of maturity modeling. Part 2: Alternative models and sensitivity analysis. $A A P G$ Bullettin, 76, 47-66.

Xiang, E., Guo, R., Dosso, S. E., Liu, J., Dong, H., \& Ren, Z. (2018). Efficient hierarchical trans-dimensional Bayesian inversion of magnetotelluric data. Geophysical Journal International, 213, 1751-1767. https://doi.org/10.1093/gii/ggy071 